\title{
Scaling of internal dissipation of polycrystalline solids on grain-size and frequency
}

\author{
Chuangchuang Duan a,b, Yujie Wei ${ }^{\mathrm{a}, \mathrm{b}, *}$ \\ ${ }^{a}$ LNM, Institute of Mechanics, Chinese Academy of Sciences, Beijing 100190, China \\ ${ }^{\mathrm{b}}$ School of Engineering Sciences, University of Chinese Academy of Sciences, Beijing 100049, China
}

\section{A R T I C L E I N F O}

\section{Article history:}

Received 4 May 2020

Revised 2 October 2020

Accepted 2 October 2020

Available online 12 October 2020

\section{Keywords:}

Internal friction

Loss modulus

Dissipation

Viscoelasticity

Polycrystalline solids

\begin{abstract}
A B S T R A C T
Internal friction is essential for nearly all solids to dissipate kinetic energy through internal mechanisms. The pioneering analyses by Zener (Phys. Rev. 60 (1941) 906-908) and Kê (Phys. Rev. 71 (1947) 533-546) demonstrated the existence of a single friction peak in the loss modulus spectrum of polycrystalline solids, which is attributed to viscous sliding in grain boundaries. In this study, we establish a continuum model coupled elastic deformation, viscous creep and diffusion in grain boundaries and reveal the existence of a second loss modulus peak resulted from viscous deformation within grain boundaries. The corresponding two frequencies when internal dissipation reaches its local maximum depend on grain size $d$, one is inversely proportional to $d$, and the other is proportional to $d^{-3}$. The effects of local elasticity and diffusion in grain boundaries on the loss modulus spectrum are examined and the condition when the two peaks emerge is identified. The findings can be applied to granular and porous materials, and complex rheology in geosciences, where internal dissipation is momentous for waves and seismic activities.
\end{abstract}

(c) 2020 Acta Materialia Inc. Published by Elsevier Ltd. All rights reserved.

\section{Introduction}

Internal dissipation or internal friction in solids is ubiquitous and occurs in most parts of dynamic systems like cars, planes, musical instruments, bridges, skyscrapers, and so on. The dissipation at the macroscopically structural level can be well understood. At the microscopic level, heterogeneity in microstructures is the primary source of internal dissipation in materials. Collective microscopic deformation in those weak zones and interfaces gives rise to the dissipative behavior of solids we observe in the macroscopic scale. The pioneering research by Zener [1] who considered grain boundary (GB) relaxation as the damping source in polycrystalline solids was successful in predicting the relaxation modulus of polycrystalline materials, and suggested the existence of a maximum internal friction in frequency space due to slippery GBs, which later on was verified by Kê [2] in polycrystalline aluminum. Subsequently, more experiments confirmed the internal friction peak resulted from GB relaxation in polycrystalline metals [3-10]. Because internal dissipation in frequency or temperature space is associated with microscopic motions of particular defects of different size [11], the concept has also been implemented in DMA dynamic mechanical analysis, and is broadly employed to identify

\footnotetext{
* Corresponding author.
}

activation time and energy scales of defects in advanced materials, from crystalline metals [12,13], metallic glasses [14-16], to polymeric materials $[17,18]$.

The significance of internal dissipation is not limited to engineering materials. In the field of geoscience, the mantle is said to behave as an elastic solid in temporal scale from 1 to $10^{4}$ seconds but behaves as a viscous fluid if observing in a time window of $10^{11}$ to $10^{17}$ seconds [19]. The deformation of subduction zones is controlled by both the short-term and long-term viscous behavior of the mantel. Viscoelastic relaxation was included to reassess the physic of the subduction earthquake cycle and predict results that are consistent with the observations [20]. There is experimental evidence that the attenuation of seismic waves is correlated with viscous sliding between weak zones and faults as well [21-23]. A detailed understanding of the coupling of viscous and elastic deformation and its related time scales will allow better interpretation of seismological data [24,25].

Despite the significant progress on understanding the origin of internal dissipation, there are growing interests to build up connections among macroscopic dissipation with micro or nanoscale deformation mechanisms. Ghahremani [26] and Lee and Morris [27] analyzed the effect of viscous GB sliding on anelasticity of polycrystalline materials and obtained a single Debye peak in the loss modulus spectrum. In the studies of Morris and Jackson [28] and Lee et al. [29], GB diffusion and viscous slip with distinct 
time scales were considered as two mechanisms of energy dissipation. Besides the single peak resulted from GB sliding, a broad region showing mildly increasing internal friction with decreasing frequency at the low-frequency regime was found in the internal friction spectrum. It is interesting to note, however, there are experimental reports on two internal dissipation peaks related to GB relaxation in ultra-fine grained polycrystalline materials [3032] but the existing analyses [1,26-29] don't predict two peaks. Such inconsistency may arise from the standard treatments that model GB as an interface and do not account for the viscous or elastic deformation in the normal direction of GB in the aforementioned theories and simulations. As grain size decreases down to nanometers, GB volume fraction becomes important, and treating $\mathrm{GBs}$ as zero-thickness interfaces is no longer valid. In this study, we consider GBs have small yet non-zero thickness, which may accommodate deformation in both their tangential and normal directions. These two deformation modes are indispensable to capture the dynamic mechanical response of those solids when grains fall within the nanoscale [33].

We perform micromechanical finite element calculations to investigate internal dissipation aroused from GB relaxation in polycrystalline materials over a broad frequency range. GBs are treated as continuum media and are modeled using continuum elements instead of interface elements. We present the theoretical formulations and micromechanical approach in Section 2. Following the analyses by Zener [1], Ghahremani [26] and Lee and Morris [27], we study internal dissipation as a result of viscous deformation in GBs in Section 3. In Section 4, we examine the role of GB elasticity and GB diffusion in the loss modulus spectrum and identify the condition when the two peaks emerge. We conclude in Section 5 with final remarks and discussions.

\section{Micromechanical modelling}

\subsection{The continuum theory}

It is expected that GB can relax much more quickly than grain interior does, thus in the time scale of GB relaxation, grain interior is assumed to be linear and isotropically elastic with bulk modulus $K$ and shear modulus $G$.

Without loss of generality, we follow the treatment employed by Zener [1], Ghahremani [26] and Lee and Morris [27] and consider relaxation aroused from viscous GBs. However, our model differs from the previous models in two ways: In the models of Ghahremani [26] and Lee and Morris [27], GB was taken as an interface and across which the normal component of the displacement vector was continuous. In the analysis of Zener [1], the normal stress across GBs doesn't relax. In this paper, we account for the two missing physical mechanisms: (1) GBs are of small yet finite thickness, and (2) normal stress relaxation within GBs is involved. We adopt continuum theory to take elastic deformation, viscous creep and diffusion in GBs into account. The formulations of the model are given as follows.

(1) Force balance: $\sigma_{i j, j}=0$, where, $\sigma_{i j}$ is the Cauchy stress, and a comma before a subscript indicates partial differentiation with respect to coordinates $x_{i}$ or $x_{j}, i, j=1,2,3$.

(2) Diffusion mechanism: mass conservation requires that the divergence of the diffusion flux $\boldsymbol{J}$ must be accommodated by deformation [34] and it gives

$\dot{\varepsilon}_{k k}+\Omega J_{k, k}-\frac{\dot{\sigma}_{h}}{K}=0$,

where, a repeated subscript implies summation over $k$ from 1 to 3; a dot above a variable denotes its partial differentiation with respect to time, $\dot{\varepsilon}_{i j}$ is the strain rate, $\dot{\varepsilon}_{k k}$ is the volumetric strain rate; with $\Omega$ being the atomic volume, $\Omega J_{k, k}$ is volumetric rate of diffusion, and $\sigma_{h}=\sigma_{k k} / 3$ is the hydrostatic stress and $K$ is the bulk modulus. The bulk modulus of GBs is taken to be the same as that of grain interiors considering volumetric deformation contributes negligible relaxation [35].

(3) The constitutive law for diffusion: The diffusion flux $\boldsymbol{J}$ is proportional to the gradient of a stress-dependent chemical potential $\mu[36]$ :

$J_{i}=-\frac{D}{k_{\mathrm{B}} T \Omega} \mu_{, i}$,

where $D$ is the diffusion coefficient, $k_{\mathrm{B}}$ Boltzmann's constant, and $T$ the absolute temperature. The chemical potential $\mu$ relates to the hydrostatic stress $\sigma_{h}$ as

$\mu=\mu_{0}-\Omega \sigma_{h}$,

where, $\mu_{0}$ is the reference potential. Inserting Eqs. (2) and (3) into Eq. (1), the diffusion equation can be reformulated as

$\dot{\varepsilon}_{k k}+\frac{D \Omega}{k_{B} T} \sigma_{h, k k}-\frac{\dot{\sigma}_{h}}{K}=0$.

(4) The constitutive equation: The deviatoric stress $\sigma_{i j}^{\prime}$ is related to the deviatoric strain $e_{i j}$ and its rate $\dot{e}_{i j}$ by

$\sigma_{i j}^{\prime}=2 \eta \dot{e}_{i j}+2 G_{R} e_{i j}$, with $e_{i j}=\varepsilon_{i j}-\frac{1}{3} \varepsilon_{k k} \delta_{i j}$,

where $\eta$ and $G_{R}$ are the viscosity and shear modulus for GB materials. Note we use a shear modulus to represent resilient deformation in GBs, with $G_{R} \rightarrow 0$ for fluidic GBs and $G_{R} \rightarrow G$ for indistinguishable GBs from grain interiors.

Eq. (5) may be reformulated in terms of Cauchy stress tensor as

$\sigma_{i j}=2 \eta\left(\dot{\varepsilon}_{i j}-\frac{1}{3} \dot{\varepsilon}_{k k} \delta_{i j}\right)+2 G_{R}\left(\varepsilon_{i j}-\frac{1}{3} \varepsilon_{k k} \delta_{i j}\right)+\sigma_{h} \delta_{i j}$

Substituting Eq. (6) into the force balance equation, we obtain

$2 \eta\left(\dot{\varepsilon}_{i j}-\frac{1}{3} \dot{\varepsilon}_{k k} \delta_{i j}\right)_{, j}+2 G_{R}\left(\varepsilon_{i j}-\frac{1}{3} \varepsilon_{k k} \delta_{i j}\right)_{, j}+\sigma_{h, i}=0$.

The continuum theory described above can be implemented in commercial FE package ABAQUS [37] as a user-element by including the hydrostatic stress as an additional unknown variable [38]. Since the governing Eqs. (4) and (7) are linear, a dimensionless parameter that characterizes the relative rate of local diffusion and viscous creep is obtained [34,39]

$R=\frac{\eta D \Omega}{k_{\mathrm{B}} T d^{2}}$

where, $d$ is the grain size. When $R=1$, the local deformation rate of diffusion and creep are comparable; for $R \ll 1$, the deformation rate by diffusion is far slower than that by viscous creep.

\subsection{Model description}

Representative samples consist of hexagonal grains for twodimensional (2D) calculations and Wigner-Seitz cells for threedimensional (3D) simulations are employed to investigate the dynamic properties of polycrystalline solids. As illustrated in Fig. 1, the grain interiors and GBs are green and red, respectively. Such highly symmetric structures enable us to apply periodic boundary conditions and realize theoretically infinite samples. We describe the detailed procedures on how to realize periodic boundary conditions in 2D hexagonal grains and Wigner-Seitz cells in Appendix A, in turn. The enlarged region in Fig. 1 shows the mesh in the GBs. We implement the constitutive behavior for GB materials by developing plane-strain, four-node quadrilateral elements for 2D modelling and eight-node brick elements for 3D modelling. For 
(a)

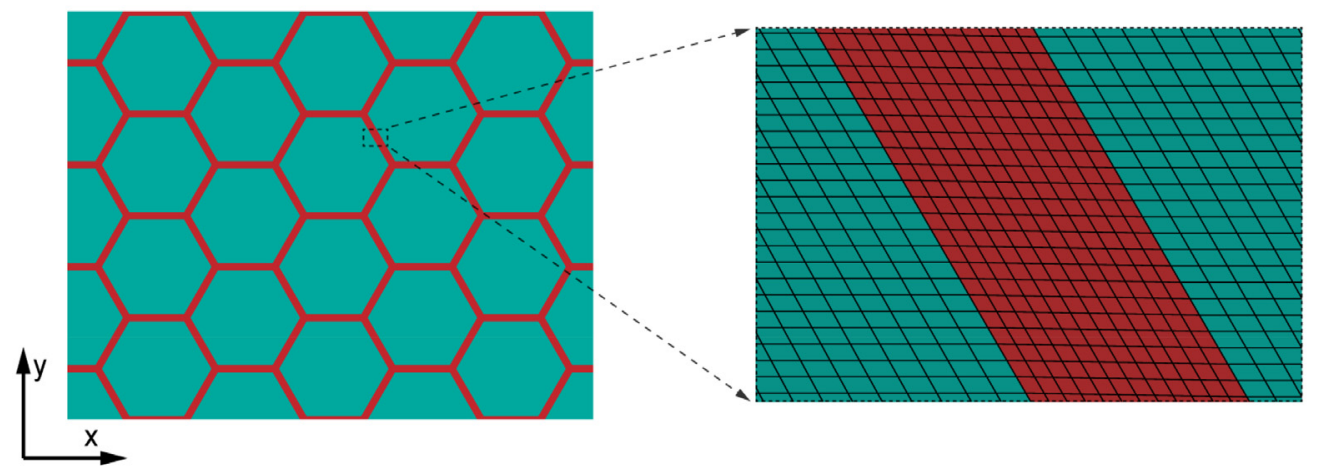

(b)
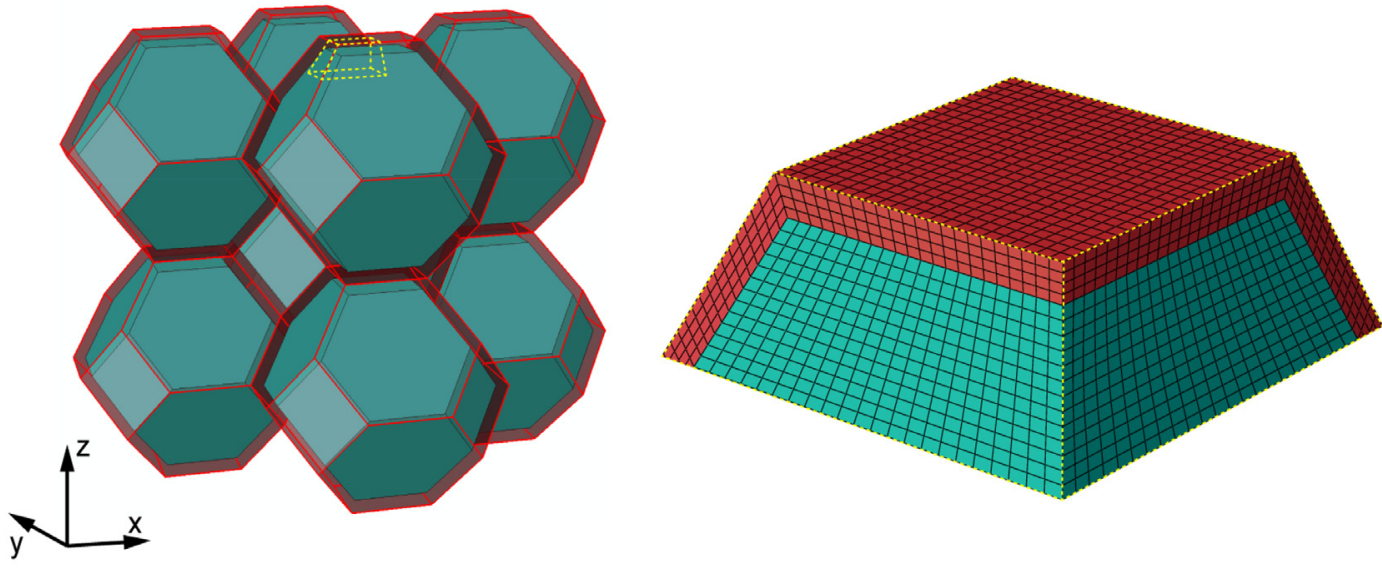

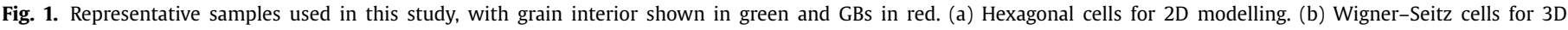

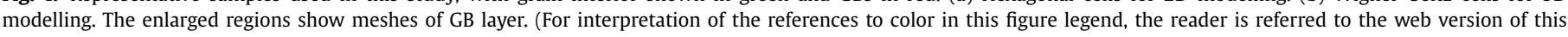
article.)

grain interiors, we adopt built-in elements in ABAQUS [37], C3D8 for 3D models and CPE4 for 2D models.

For a polycrystalline sample with grain size $d$ and GB thickness $t$, we obtain volume fraction $\phi$ of GB materials as $\phi=\alpha t / d$, where $\alpha=2 \sqrt{2 \sqrt{3} / \pi}$ for 2D hexagonal grains and $\alpha=3 \sqrt[3]{3 / \pi}$ for 3D Wigner-Seitz cells. The grain size $d$ is taken as the diameter of a sphere whose volume equals to that of the Wigner-Seitz cell for the 3D cases, and by the same token $d$ as the diameter of an equivalent circle for a hexagon in 2D samples.

\subsection{Calculation of dynamic modulus}

When a polycrystalline solid is subject to cyclic strain, the local stresses and strains in grain interiors remain in phase, while in GBs they are out of phase. Therefore, the macroscopic stresses and strains are in general out of phase. Complex modulus or dynamic modulus is therefore introduced to characterize the dynamic properties of the polycrystalline aggregates.

For the Wigner-Seitz model shown in Fig. 1b, we apply a timedependent displacement in the $z$ direction, $w=w_{0} \sin (\omega t)$, correspondingly we have a cyclic macroscopic strain $\bar{\varepsilon}=\varepsilon_{0} \sin (\omega t)$. A typical load history of applied macroscopic strain adopted in the simulation is shown in Fig. $2 \mathrm{a}$ (red line). The resultant macroscopic stress $\bar{\sigma}$ from FE simulations (blue open circles in Fig. 2a) is also a sinusoidal function of time with the same frequency $\omega$ after a few cycles. By fitting the calculated results (blue line in Fig. 2a), we can express the macroscopic stress in the form of $\bar{\sigma}=\sigma_{0} \sin (\omega t+\theta)$, where $\theta$ is the phase shift between the macroscopic stress and strain. The effective complex Young's modulus $E^{*}$ (or shear mod- ulus $G^{*}$ if a cyclic shear strain is applied) can be obtained by definition,

$E^{*}(\omega)=\hat{E} e^{i \theta}=E^{\prime}(\omega)+i E^{\prime \prime}(\omega)$,

where $\hat{E}=\sigma_{0} / \varepsilon_{0}$ is the magnitude of the complex modulus. The complex modulus can be decomposed into two parts, $E^{\prime}=\hat{E} \cos (\theta)$ is the storage modulus and $E^{\prime \prime}=\hat{E} \sin (\theta)$ is the loss modulus which quantifies energy dissipation. In this way, the effective complex Young's modulus of the sample can be calculated.

From Eqs. (4) and (7), we can see that the non-dimensional complex Young's modulus $E^{*} / E$ (where $E=\frac{9 K G}{3 K+G}$ is the Young's modulus of grain interiors) depends on Poisson's ratio of grain interiors $v$, GB volume fraction $\phi$ (grain size), the ratio of shear moduli of GBs and grain interior $G_{R} / G$, and the dimensionless parameter $R$ and the non-dimensional frequency $\omega \eta / G$. In this paper, we investigate the loss modulus as a function of frequency and how the viscous deformation in GBs contribute to the loss modulus.

\section{Internal dissipation and grain size}

\subsection{The two peaks phenomenon}

In order to investigate the contribution of viscous deformation in GBs to the loss modulus, we consider first the special case of $R=0$ and $G_{R} / G=0$. The effective complex modulus is then calculated over a broad frequency range for the Wigner-Seitz models with $v=0.3$ and different $\phi$. Fig. $2 \mathrm{~b}$ and c show its magnitude $\hat{E}$ and phase angle $\theta$, respectively. As seen in Fig. $2 \mathbf{b}$, the value of complex modulus $\hat{E} / E$ increases with the dimensionless frequency 
(a)

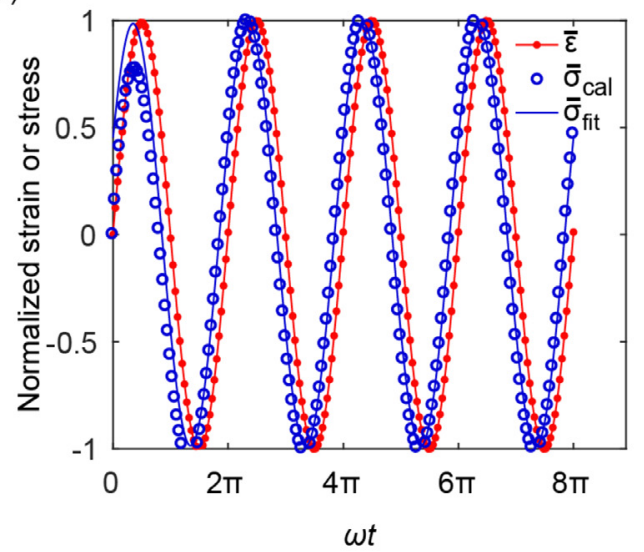

(c)

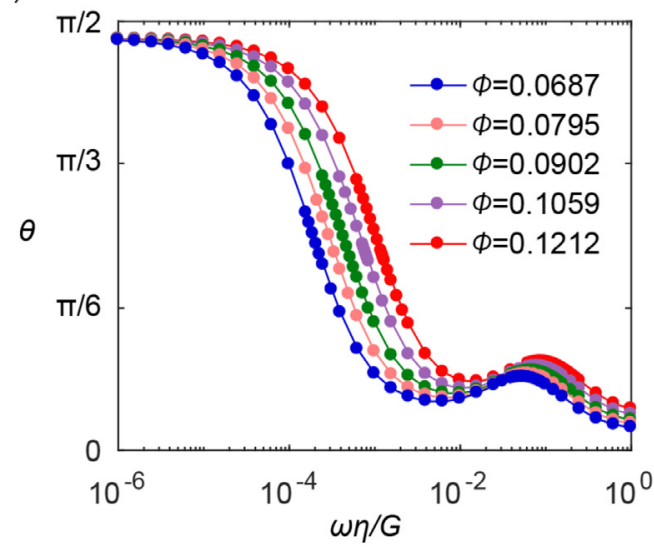

(b)

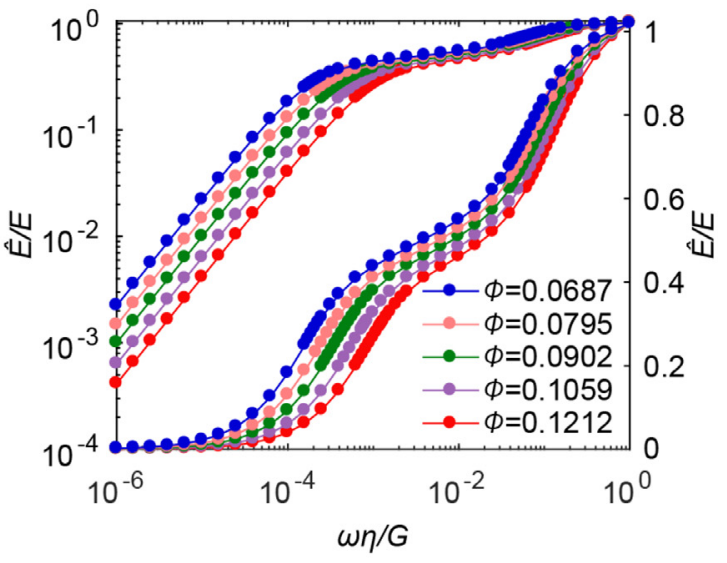

(d)

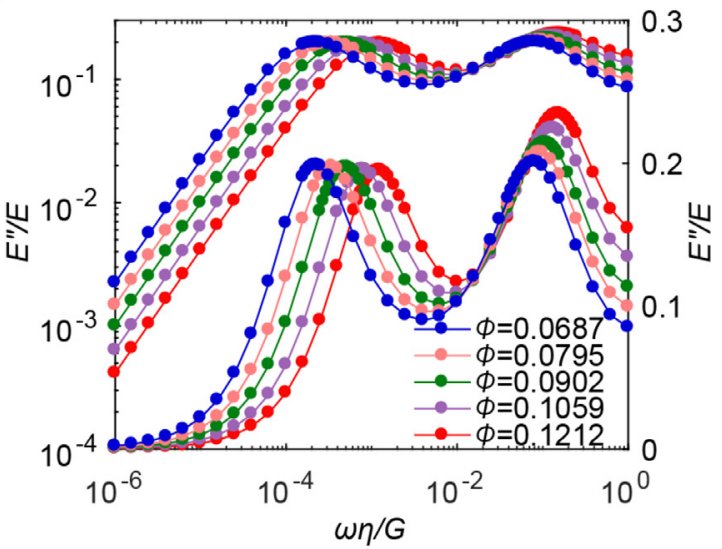

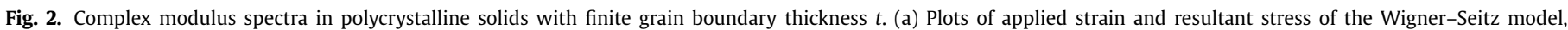

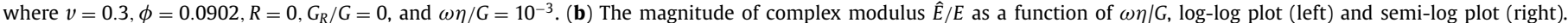
(c) The phase angle $\theta$ of complex modulus versus $\omega \eta / G$. (d) Loss modulus spectra, log-log plot (left) and semi-log plot (right).

$\omega \eta / G$ due to relaxation in GBs. As $\omega \eta / G$ decreases, phase angle $\theta$ maximizes at a frequency characterized by the point of the fastest decreasing rate of $\hat{E} / E$ on $\omega \eta / G$. Note when $\omega \eta / G$ approaches to $0, \hat{E} / E$ is proportional to $\omega \eta / G$ and $\theta$ converges to $\pi / 2$. Therefore, in the low frequency end, deformation in the polycrystalline solid is dominated by viscous creep in the GBs, and the macroscopic behavior of the polycrystalline sample can be represented by a Maxwell model and is non-recoverable [11].

Fig. 2d shows the loss modulus $E^{\prime \prime} / E$ as a function of $\omega \eta / G$, and here we see two well-separated peaks. It can be identified that each peak in the loss modulus spectra corresponds to the quickest reduction of complex modulus in Fig. $2 \mathrm{~b}$ when $\omega \eta / G$ decreases. We refer the two peaks as $P_{1}$ and $P_{2}$, and their corresponding frequencies are $\omega_{1}$ and $\omega_{2}$, respectively, and $\omega_{1}>\omega_{2}$. It can be seen from Fig. $2 \mathrm{~d}$ that both $\omega_{1}$ and $\omega_{2}$ increase with $\phi$, i.e. they increase with decreasing grain size. In general, the non-dimensional loss modulus is a function of $\omega \eta / G, \phi, G_{R} / G, R$ and $v$. Therefore, the critical frequencies at which loss modulus maximizes depend on $\phi, G_{R} / G, R$ and $v$ as well. Here, we focus on the dependency of $\omega_{1}$ and $\omega_{2}$ on $\phi$.

\subsection{The scaling law of critical frequencies on grain size}

The scaling behavior of the critical frequencies on GB volume fraction $\phi$ is shown in Fig. 3. From Fig. 3a, we see that $\omega_{1}$ is proportional to $\phi, \omega_{1} \sim G \phi / \eta$, and $\omega_{2}$ is proportional to $\phi^{3}$, $\omega_{2} \sim G \phi^{3} / \eta$. The loss modulus spectra in Fig. 2d suggests that the amplitude $h_{2}$ of the second peak $P_{2}$ is nearly independent of $\phi$, while the amplitude $h_{1}$ at $P_{1}$ increases with $\phi$, as demon- strated in Fig. 3b, and $h_{1}$ follows a linear relationship with $\phi$, $h_{1}=0.6401 \phi+0.1571$. When $\phi$ goes to zero, $h_{1}$ approaches to 0.1571 for Wigner-Seitz cells. This value is comparable with the prediction of 0.1898 by Zener [1] for the special case when $\phi=0$ and for ideally spherical grains, other than polyhedrons we used here.

We also note, as seen in Fig. 2d, the loss modulus with fixed $\omega \eta / G$ decreases with $\phi$ when $\omega \eta / G$ is small, but increases with $\phi$ for large $\omega \eta / G$. We adopt a dimensionless loss modulus $E^{\prime \prime} \mid \omega \eta$, which measures the effective viscosity of the polycrystalline sample. The dependence of $E^{\prime \prime} \mid \omega \eta$ on $\phi$ is shown in Fig. $3 c$ and d, corresponding to the cases of $\omega \eta / G=1$ and $10^{-6}$, respectively. We see $E^{\prime \prime} \mid \omega \eta$ increases linearly with $\phi$ when $\omega \eta / G=1$, but is proportional to $\phi^{-3}$ if $\omega \eta / G=10^{-6}$. The striking difference in the dependence of the effective viscosity on $\phi$ for different $\omega \eta / G$ demonstrates that the governing relaxation mode in polycrystalline aggregates is contingent on $\omega \eta / G$.

\subsection{Physical origin of the two internal dissipation peaks}

The underlying mechanisms accounting for the scaling behavior is elucidated through an assembly of two-dimensional (2D) hexagonal grains, which is physically straightforward without loss of generality, as shown in Fig. 4a. For small $\omega \eta / G$, grain interiors is more rigid than viscous GBs. We examine the response of GBs of different orientations in the hexagonal model when pure shear is applied, as seen in Fig. 4a: The GBs $A-O$ (between grains $A$ and $O$ ) and $D-O$ undergo pure shear as well. Hence the traction $F_{S}$ along the tangential of GBs $A-O$ and $D-O$ in terms of the rela- 
(a)

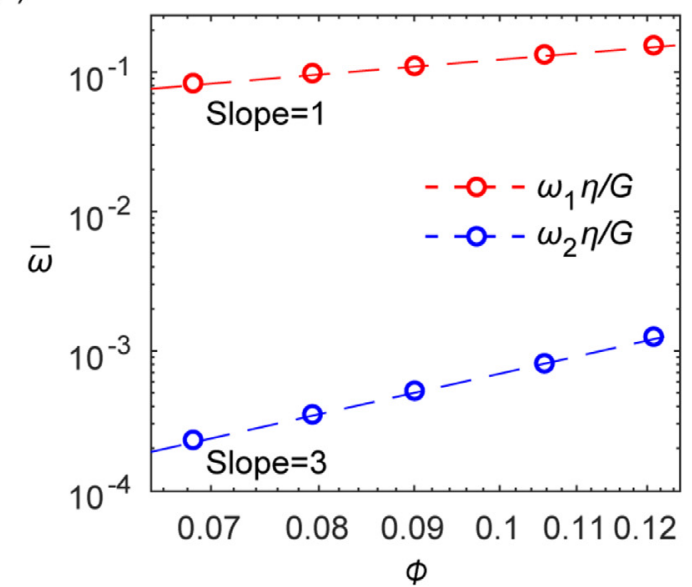

(c)

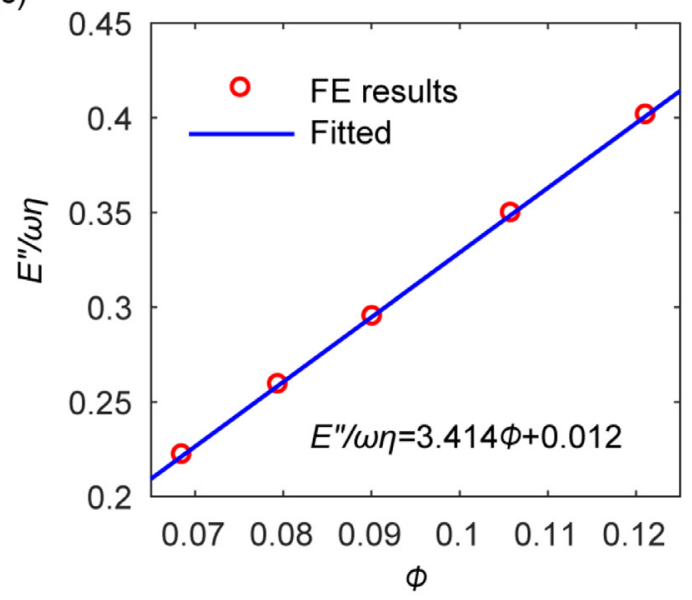

(b)

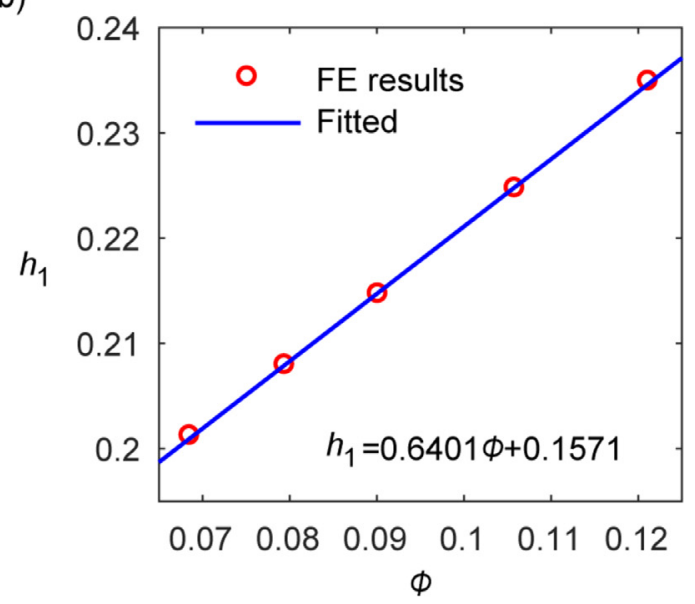

(d)

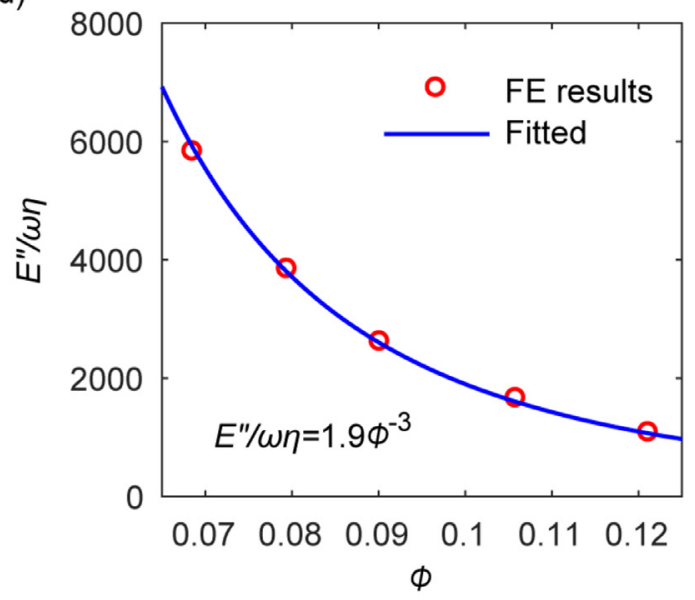

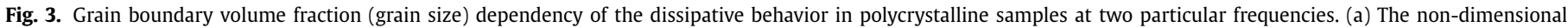

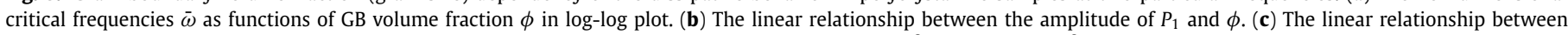
non-dimensional effective viscosity $E^{\prime \prime} \mid \omega \eta$ and $\phi$ when $\omega \eta / G=1$. (d) $E^{\prime \prime} \mid \omega \eta$ linearly depends on $\phi^{-3}$ for $\omega \eta / G=10^{-6}$.

tive shearing velocities $V_{s}$ is obtained as $F_{S}=\eta \frac{l}{t} V_{s}=\frac{2}{\sqrt{3}} \frac{\eta}{\phi} V_{s}$, where $l$ is GB length and the out of plane dimension is taken to be unit. Now we consider the scenario when grains $C$ and $F$ move away from grain $O$ while grains $B$ and $E$ move toward grain $O$ for compatible deformation (see Fig. 4a): it leads to GB deformation in its normal direction with GBs $C-O$ and $F-O$ being dilated but GBs $B-O$ and $E-O$ being squeezed [40]. The normal traction $F_{n}$ is deduced from the classical lubrication theory [41] and is related to the GB normal velocity $V_{n}, F_{n}=\eta\left(\frac{l}{t}\right)^{3} V_{n}=\frac{8}{3 \sqrt{3}} \frac{\eta}{\phi^{3}} V_{n}$. The tangential and normal deformation modes of GBs are sketched in Fig. $4 \mathrm{~b}$. Thus, we have two effective viscosities corresponding to the two deformation modes,

$\eta_{1}=\frac{\mathrm{a}}{\phi} \eta$ and $\eta_{2}=\frac{\mathrm{b}}{\phi^{3}} \eta$,

where $\eta_{1}$ and $\eta_{2}$ represent respectively resistance to shear and to normal deformation, and $a$ and $b$ are two constants. When the loading frequency $\omega$ approaches to $\frac{G}{\eta_{1}}=\frac{\phi G}{a \eta}$, the tangential deformation (sliding) will give rise to a loss modulus peak as previously suggested [1,2,26-29,35]. Likewise, when $\omega$ is close to $\frac{G}{\eta_{2}}=\frac{\phi^{3} G}{b \eta}$, deformation in the normal direction will lead to another loss modulus peak. Since $\phi$ is small, the two peaks are well separated in the frequency space, as seen in Fig. $2 d$.

The complex physical mechanism can be conceptually captured by two parallel Maxwell models shown in Fig. $4 \mathrm{~b}$. Note the two springs represent contribution from the grains, and we assume $k_{1}=c G$ and $k_{2}=e G$, where $c$, $e$ are constants. Hence, the phenomenological model in Fig. $4 \mathrm{~b}$ leads to a complex shear modulus $G^{*}$ :

$G^{*}=1 /\left(\frac{1}{k_{1}}+\frac{1}{i \omega \eta_{1}}\right)+1 /\left(\frac{1}{k_{2}}+\frac{1}{i \omega \eta_{2}}\right)$

The loss shear modulus $G^{\prime \prime}$ of the system is given as:

$\frac{G^{\prime \prime}}{G}=\frac{c^{2}}{a} \frac{\phi(\omega \eta / G)}{\left(\frac{c}{a} \phi\right)^{2}+(\omega \eta / G)^{2}}+\frac{e^{2}}{b} \frac{\phi^{3}(\omega \eta / G)}{\left(\frac{e}{b} \phi^{3}\right)^{2}+(\omega \eta / G)^{2}}$

According to Eq. (12), there are two critical frequencies at which the loss modulus peaks: $\omega_{1}=\frac{c}{a} \phi G / \eta$ and $\omega_{2}=\frac{e}{b} \phi^{3} G / \eta$, and the corresponding amplitudes of the two peaks are $h_{1}=\frac{c}{2}$ and $h_{2}=\frac{e}{2}$, respectively.

In Fig. 4c, the loss modulus $G^{\prime \prime} / G$ for the hexagonal model is plotted as a function of $\omega \eta / G$. The sample in Fig. $4 a$ is isotropic by nature and we can calculate the effective complex shear modulus of the aggregate using FE simulation. The results shown in Fig. $4 \mathrm{c}$ are for samples with $G_{R} / G=0, R=0, v=0.3$ and several $\phi$. The detailed implementation of the FE simulations can be found in Appendix A. The loss modulus spectra of the 2D samples with hexagonal grains show two separated peaks similar to those we see in the Wigner-Seitz samples. The results predicted by Eq. (12) are also plotted in Fig. 4c. The theory can capture both two peaks with consistent critical frequency at different $\phi$, and 
(a)

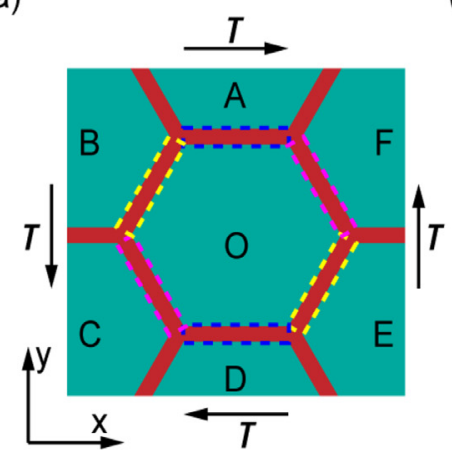

(b)

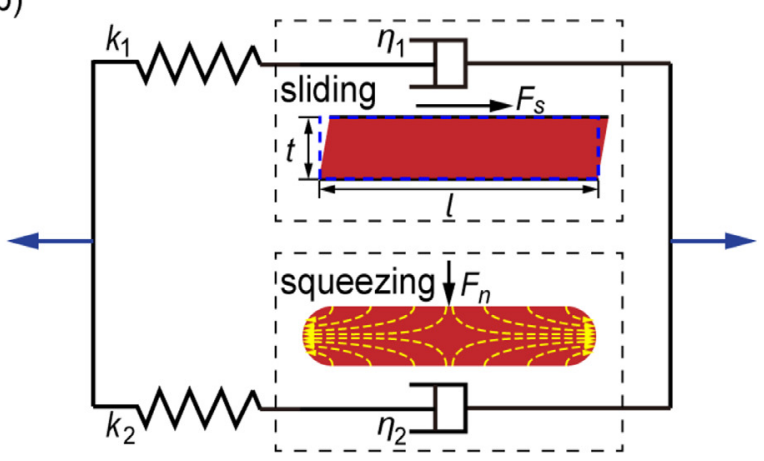

(c)

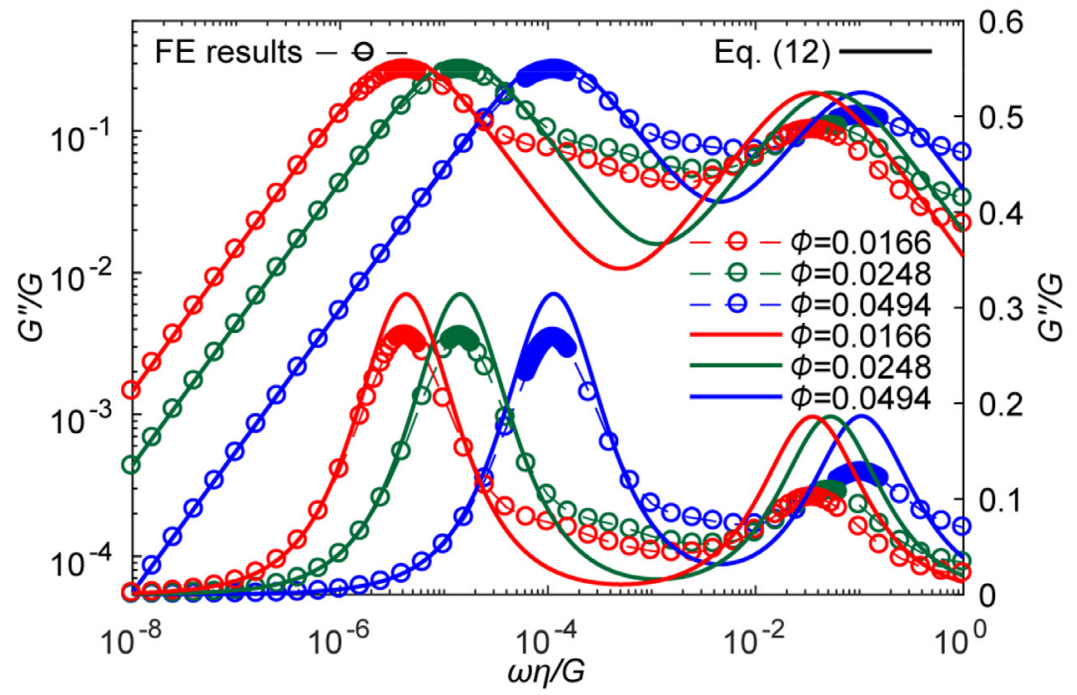

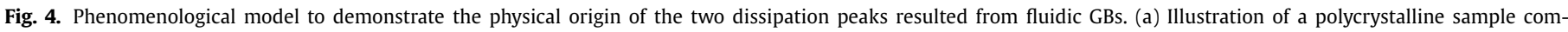

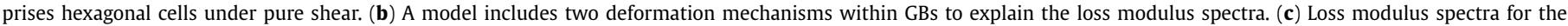

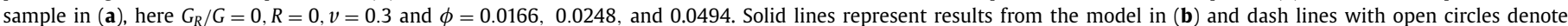
the results obtained from FE simulations.

shows excellent agreement with the results from FE analysis. The first peak $P_{1}$ can be attributed to shear relaxation across GBs as reported in previous studies [1,2,26-29,35]. The second peak $P_{2}$ results from relaxation of GB normal stresses. It is worth noting that the demonstration for the two peaks and their physical origin can be employed in many other fields. For example, the distinct relaxation model was observed in porous media [42] and was adopted by Mavko and Nur [43] to explain the intermediated time scale viscous deformation in Nankaido earthquake.

\section{The role of GB elasticity and diffusion}

\subsection{The effects of GB elasticity on the loss modulus spectrum}

Now we examine how elastic modulus of GBs affects the loss modulus spectrum and hence the scaling behavior. Fig. 5 shows the results for the Wigner-Seitz models with $v=0.3, R=0$ and $\phi=$ 0.0902 for a wide range of $G_{R} / G$. For all cases when $G_{R} / G \neq 0, \hat{E} / E$ converges to a certain value and $\theta$ is proportional to $\omega \eta / G$ when $\omega \eta / G$ approaches to zero; when $G_{R} / G=0, \hat{E} / E$ is proportional to $\omega \eta / G$, and $\theta$ approximates to $\pi / 2$. The loss modulus spectra, as seen in Fig. $5 c$, exhibit a linear regime at low frequency for all the cases of $G_{R} / G$. In Fig. $5 \mathrm{~d}$, we show the influence of $G_{R} / G$ on the loss modulus within this linear region: the loss modulus decreases with $G_{R} / G$, and decreases progressively when $G_{R} / G \approx \phi^{3}$. An important role of $G_{R} / G$ on the loss modulus spectrum is that how GB elasticity governs the existence of the peaks in a dissipation spectrum. As seen in Fig. $5 c$, when $0 \leq G_{R} / G \ll \phi^{3}$, e.g., $G_{R} / G=10^{-6}$ or $10^{-5}$, two peaks exist and the loss modulus spectrum is nearly unchanged with $G_{R} / G$; for $G_{R} / G \sim \phi^{3}$, e.g., $G_{R} / G=10^{-4}$ or $10^{-3}, P_{1}$ remain almost unchanged, but $P_{2}$ moves toward $P_{1}$ and reduces rapidly with increasing $G_{R} / G$; if $G_{R} / G$ is significantly greater than $\phi^{3}$, e.g., $G_{R} / G=10^{-2}$ or $10^{-1}$, the second peak $P_{2}$ disappears.

This dependency of the loss modulus spectrum upon $G_{R} / G$ may be captured by a modified Zener model. For $G_{R} / G>0$, the elastic and viscous deformations in GBs are coupled. Based on the Voigt type constitutive relation for GBs (see Eq. (5)), we take GB elasticity into account by adding a spring in parallel with the two dashpots in the model shown in Fig. $4 \mathrm{~b}$, and obtain the modified Zener model seen in Fig. $6 a$. For the instance of $G_{R}=0$, it represents viscous damping [11] in the modified Maxwell model. In contrast, $G_{R}>0$ in the modified Zener model gives rise to anelastic relaxation, where a continuous chain of springs will lead to a fully recoverable behavior and a rate-dependent hysteresis in the stressstrain curves. Within a loading cycle, fully recoverable deformation is realized at sufficiently long dwelling time given $G_{R}>0$. The macroscopic anelastic behavior resulted from the non-recoverable microscopic deformation is detailed in Appendix B.

The complex modulus $G^{*}$ of the modified model is then given as:

$G^{*}=1 /\left(\frac{1}{k_{1}}+\frac{1}{i \omega \eta_{1}+k_{3}}\right)+1 /\left(\frac{1}{k_{2}}+\frac{1}{i \omega \eta_{2}+k_{4}}\right)$ 
(a)

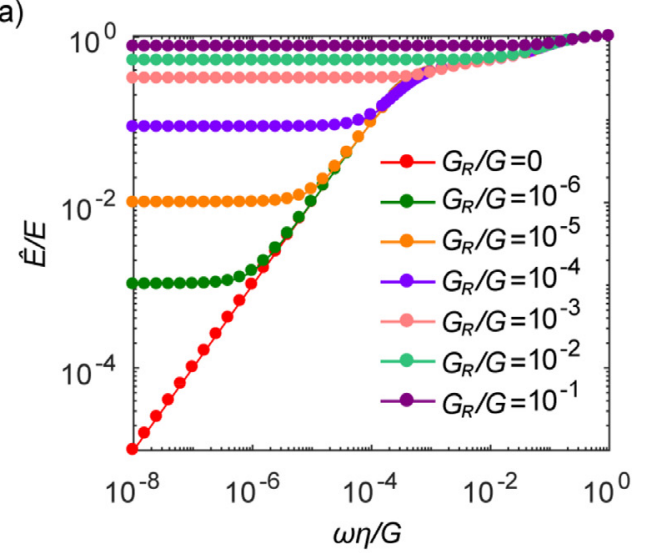

(c)

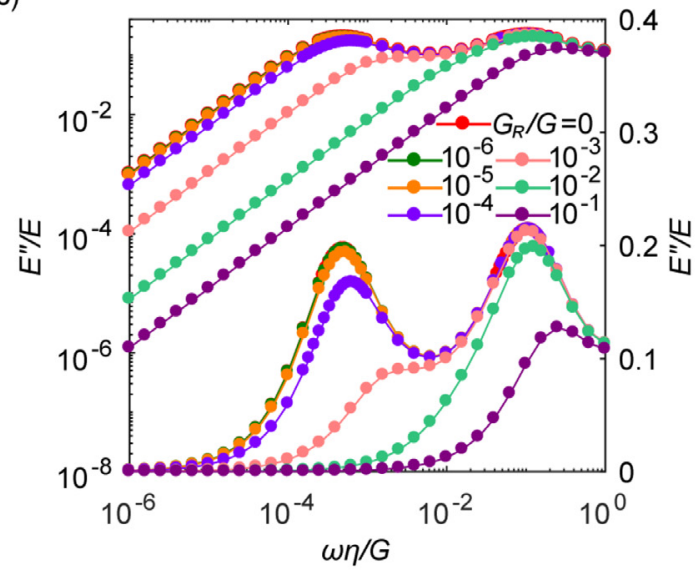

(b)

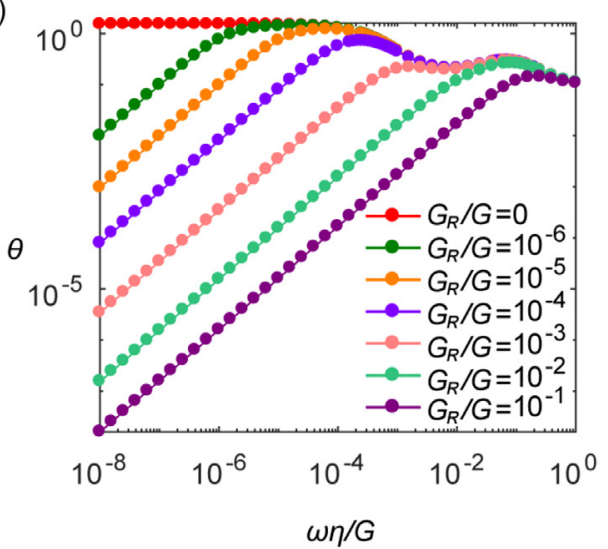

(d)

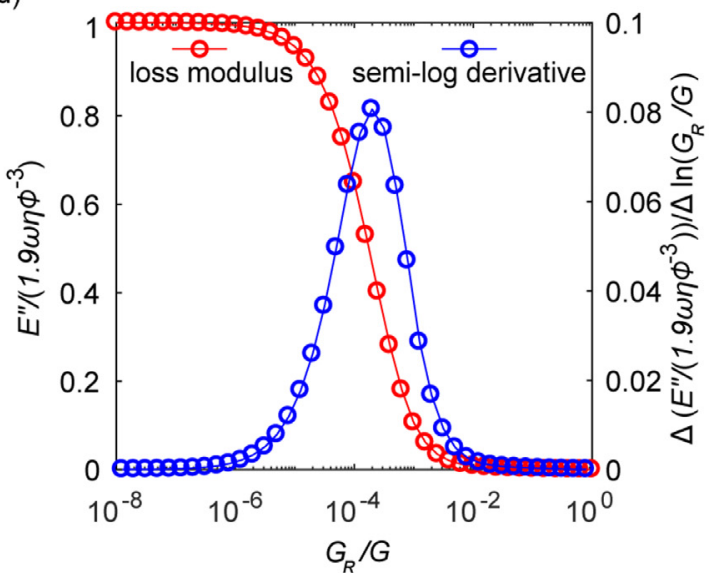

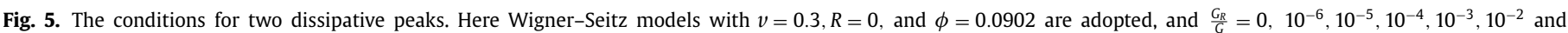

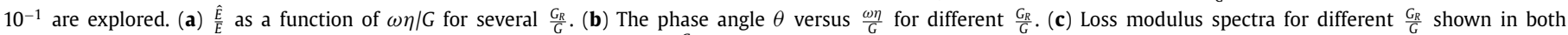
$\log -\log$ (left) and semi-log plots (right). (d) Loss modulus as a function of $\frac{G_{R}}{G}$ when $\frac{\omega \eta}{G}=10^{-6}$ (left) and its semi-log derivative (right).

(a)

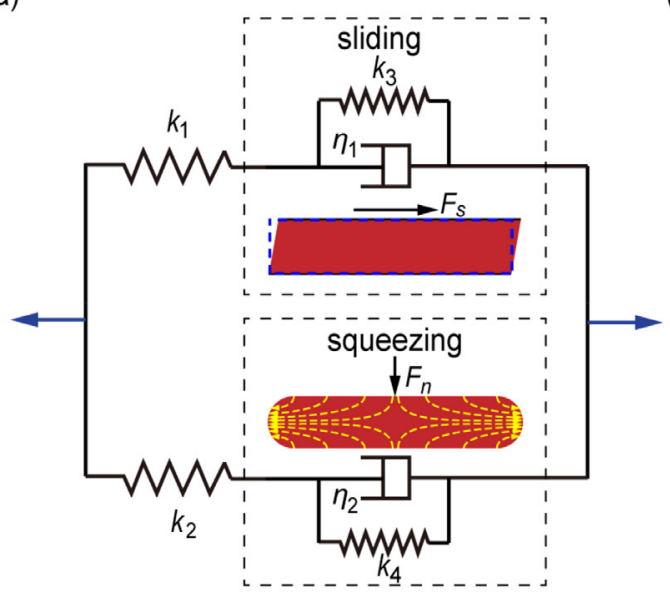

(b)

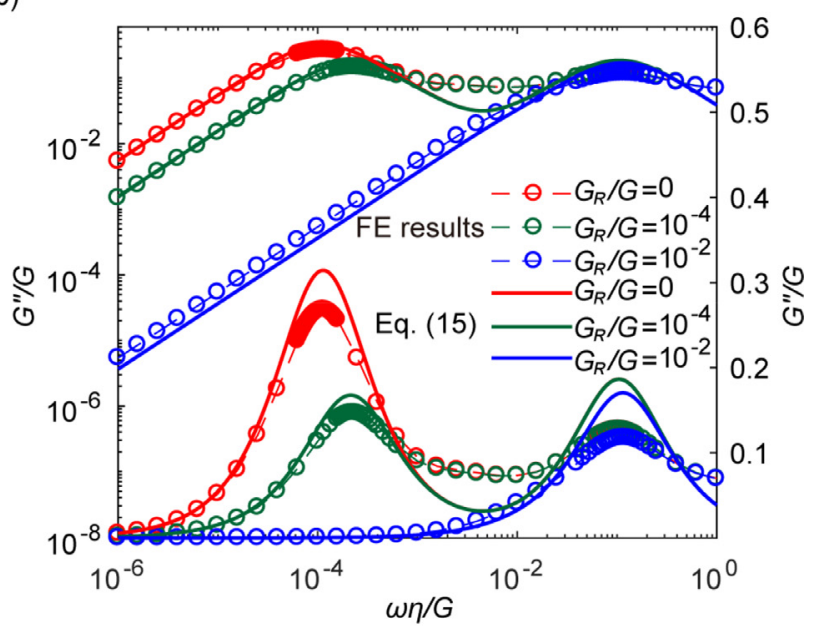

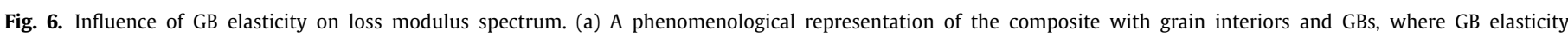

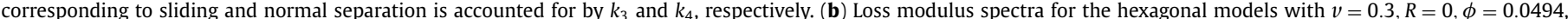
and $G_{R} / G=0,10^{-4}$ and $10^{-2}$. Here solid lines are results from the model in (a) and dash lines with open circles denote the results obtained from FE simulations.

where, $k_{1}, k_{2}, \eta_{1}, \eta_{2}$ are the same as those in the model in Fig. $4 \mathrm{~b}$, and $k_{3}$ and $k_{4}$ are introduced to include the elastic effects of GBs with non-zero $G_{R} / G$. The two spring constants $k_{3}$ and $k_{4}$ in Eq. (13) are proportional to $G_{R}$ and should also be weighted by GB volume fraction $\phi$. Their expressions can be deduced by employing the equivalence of effective complex modulus between a solid- liquid composite and a solid-solid composite [44,45]. Drawing an analogy between those two, the 'weights' of $k_{3}$ and $k_{4}$ on $\phi$ follow the same 'weights' of $\eta_{1}$ and $\eta_{2}$ on $\eta$. With Eq. (10), we obtain in sequential

$k_{3}=\frac{a}{\phi} G_{R}$ and $k_{4}=\frac{b}{\phi^{3}} G_{R}$ 
(a)

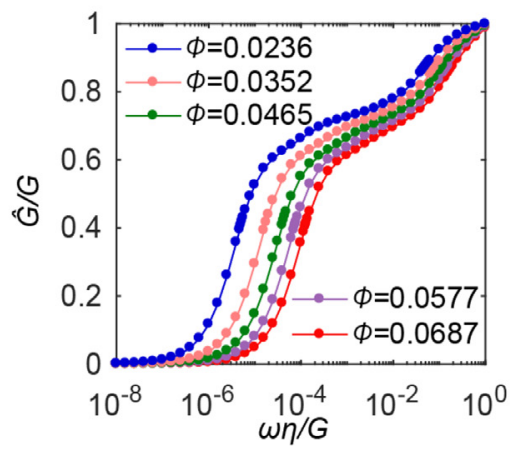

(d)

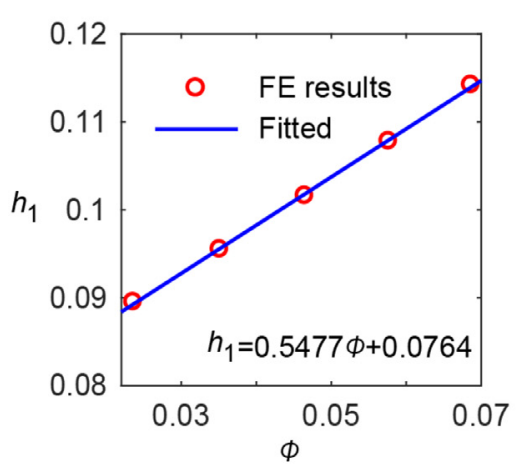

(b)

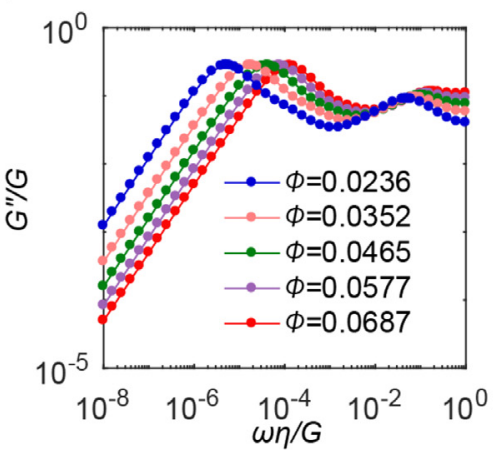

(e)

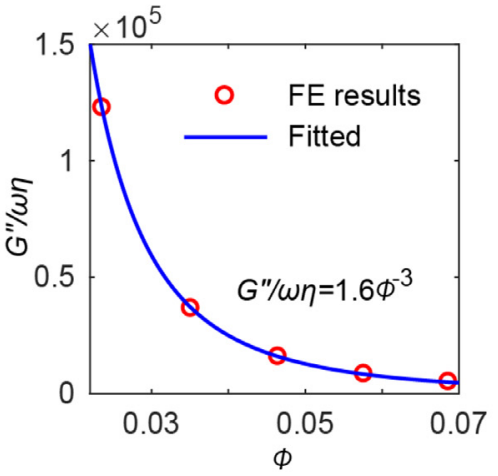

(c)

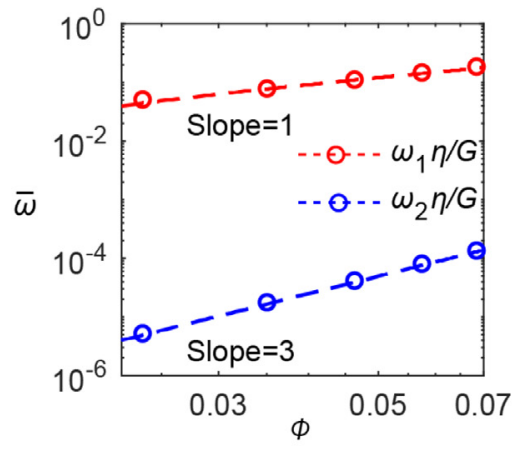

(f)

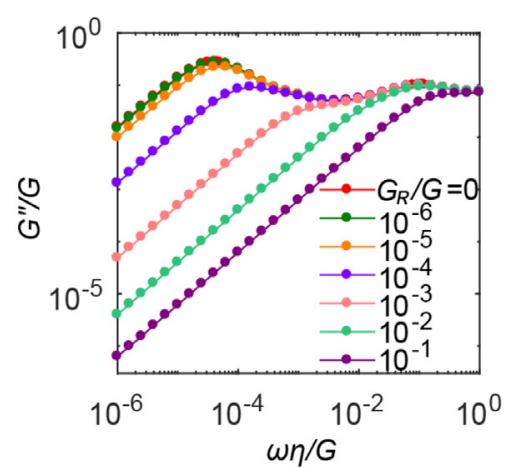

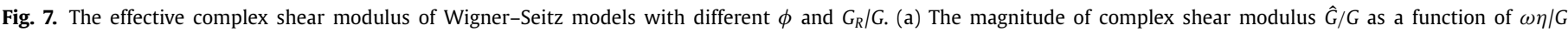

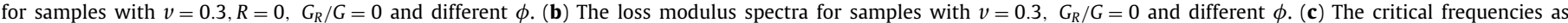

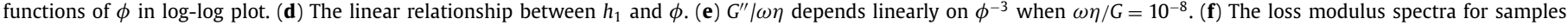
with $\phi=0.0465, v=0.3, R=0$ and different $G_{R} / G$.

With the properties for each component shown in Fig. 6a, we rewrite the loss modulus $G^{\prime \prime}$ as

$\frac{G^{\prime \prime}}{G}=\frac{c^{2}}{a} \frac{\phi(\omega \eta / G)}{\left(\frac{c}{a} \phi+G_{R} / G\right)^{2}+(\omega \eta / G)^{2}}+\frac{e^{2}}{b} \frac{\phi^{3}(\omega \eta / G)}{\left(\frac{e}{b} \phi^{3}+G_{R} / G\right)^{2}+(\omega \eta / G)^{2}}$

The two terms in the right-hand side of Eq. (15) maximize at two critical frequencies: $\left(\frac{c}{a} \phi+\frac{G_{R}}{G}\right) \frac{G}{\eta}$ and $\left(\frac{e}{b} \phi^{3}+\frac{G_{R}}{G}\right) \frac{G}{\eta}$, respectively, and the values of the associated maxima are $\frac{c^{2}}{2 a} \frac{\phi}{\left(\frac{c}{a} \phi+G_{R} / G\right)}$ and $\frac{e^{2}}{2 b} \frac{\phi^{3}}{\left(\frac{e}{b} \phi^{3}+G_{R} / G\right)}$, in turn. It is clear to see $G_{R} / G$ affects where the loss modulus maximizes and the corresponding maxima. In Fig. 6b, the loss modulus spectra for the hexagonal models with $v=0.3, \phi=0.0494, R=0$ and three typical values of $G_{R} / G$ are plotted. The results from FE simulations are in good agreement with the results obtained from the theoretical model shown in Fig. 6a.

In order to investigate the dynamic shearing response of the polycrystalline solids and verify the impact of GB elasticity on the loss modulus spectra, the effective complex shear modulus of the 3D Wigner-Seitz model is calculated. The simulation results are shown in Fig. 7. We first consider the special case when GB elasticity and GB diffusion are not involved. The complex shear modulus for models with $v=0.3, R=0, G_{R} / G=0$, and several $\phi$, is calculated. As seen in Fig. 7a, the magnitude of complex shear modulus experiences two sharp reductions with descending frequency. Correspondingly, the loss modulus maximizes at two critical frequencies, $\omega_{1} \sim G \phi / \eta$ and $\omega_{2} \sim G \phi^{3} / \eta$ (Fig. 7b, c). The amplitude of $P_{2}$ is independent of $\phi$; the amplitude of $P_{1}$, as denoted by $h_{1}$, increases linearly with $\phi$, and it follows with $h_{1}=0.5477 \phi+0.0764$ (Fig. 7d). As $\phi$ reaches to zero, $h_{1}$ approaches to 0.0764 .
Note that the BCC arrangement based Wigner-Seitz model is intrinsically anisotropic and the choice of loading directions should thus influence the response. In the simple shearing simulations using the Wigner-Seitz model (loading in the $x-z$ plane), $h_{1}$, the first peak amplitude of $\frac{G^{\prime \prime}}{G}$ (Fig. 7b), is smaller than that of $\frac{E^{\prime \prime}}{E}$ (Fig. 2d) from tension. Nevertheless, the scaling behavior remains the same for both shearing and tensile loading. In Fig. 7e, we plot the loss modulus or effective viscosity $G^{\prime \prime} \mid \omega \eta$ as a function of $\phi$ when $\omega \eta / G=10^{-8}$ (within the linear region in Fig. 7b). The effective viscosity is inversely proportional to $\phi^{3}$. The shear loss modulus spectra for Wigner-Seitz models of different GB elasticity are plotted in Fig. 7f. It can be seen that, $P_{2}$ decreases when $G_{R} / G$ increases from 0 , and it disappears when $G_{R} / G$ is several orders of magnitude greater than $\phi^{3}$. The dominant role played by GB elasticity on the dissipation spectrum is consistently observed in both cases. Similar results are obtained for the 2D hexagonal models and are also supplied in Appendix A, Fig. A.2.

\subsection{The contribution of diffusion to the loss modulus}

We now evaluate the influence of GB diffusion on the loss modulus. The complex modulus spectra are calculated for the $2 \mathrm{D}$ hexagonal models with $\phi=0.0494, v=0.3, G_{R} / G=0$ and several $R$. As can be seen from Fig. 8a, the magnitude of the complex modulus decreases faster when the diffusion rate increases. Accordingly, the loss modulus increases with increasing $R$ (Fig. 8b). An important feature of the loss modulus spectra - diffusion rate dependence is that the two peaks move closer in the frequency space with increasing $R$, and merger together when $R$ is large enough. When $R \ll \phi^{3}$ (e.g. $R=10^{-6}$ ), the loss modulus spectrum is the same as that when $R=0$. When $R$ is within the intermediate range (e.g. $R=10^{-4}, 10^{-3}$ ), the position of $P_{2}$ in the loss modulus spec- 
(a)

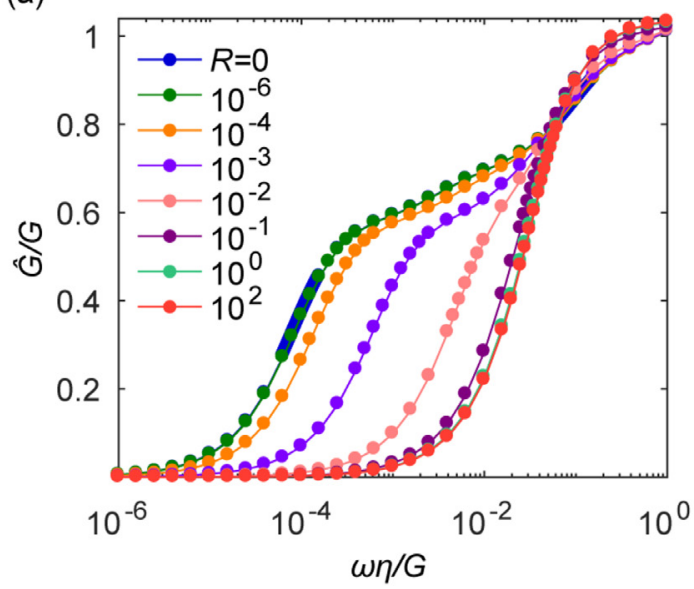

(c)

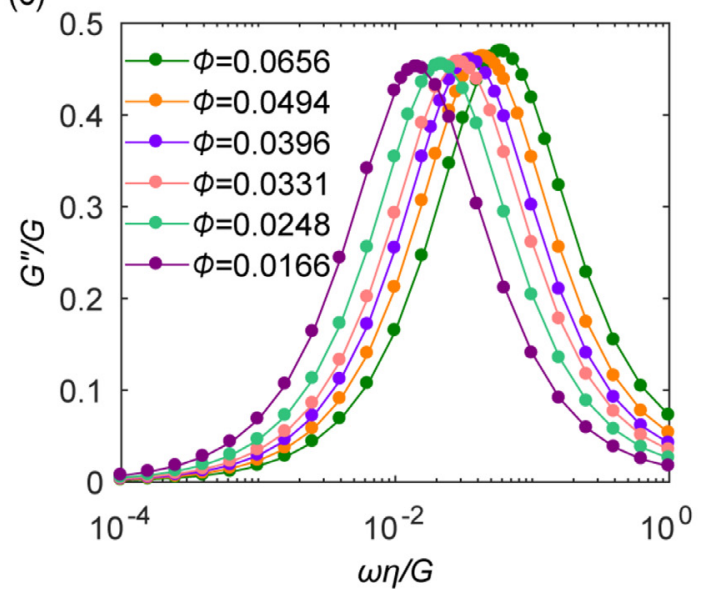

(b)

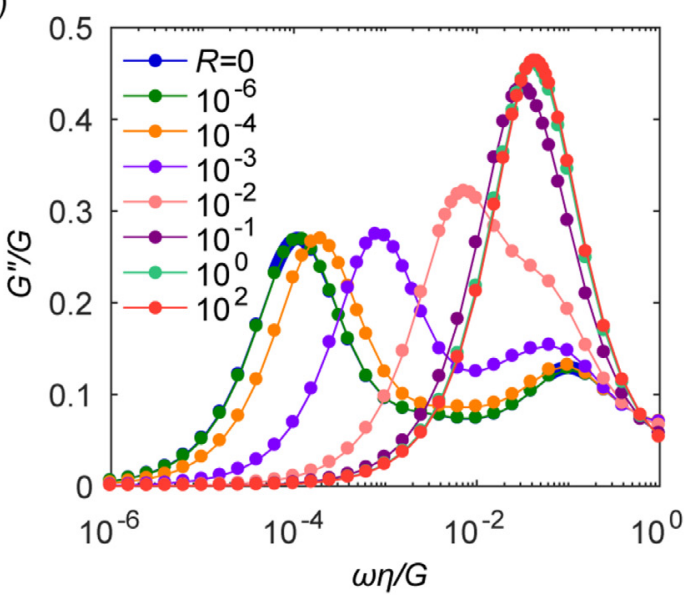

(d)

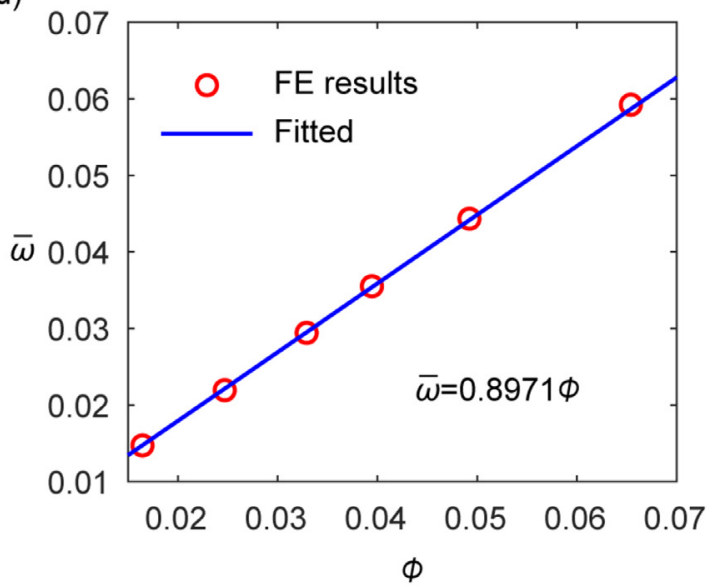

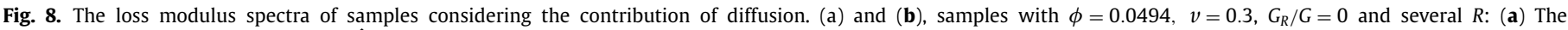

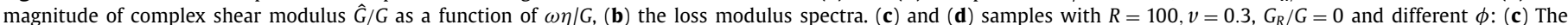
loss modulus spectra, (d) the critical frequency for the peak in (c) as a function of $\phi$.

trum is controlled by the relative rate of diffusion; the amplitude of the peak does not decrease, in contrast to the situation when $G_{R} / G$ increases. For high diffusion rates $\left(R=10^{0}, 10^{2}\right)$, the loss modulus spectrum is invariant with $R$. Fig. 8c shows the loss modulus spectra for sample with $R=100$ and different $\phi$. The critical frequency of the loss modulus peak increases with $\phi$ and is proportional to $\phi$ (Fig. 8d).

\section{Conclusions and discussions}

Plasticity in GBs may be introduced by two mechanisms, GB diffusion and GB sliding, which are broadly recognized [46,47]. Contributions from these two GB mechanisms to macroscopic plastic deformation become significant as grain sizes down to nanoscale [48-51]. GB diffusion is normally responsible for deformation in the normal direction and GB sliding in general accounts for deformation in the tangential direction. However, the ultra-high mobility of atoms in thin-layer GBs renders it fluid-like in contrast to atoms of low mobility in grain interiors. A finite and viscous layer supplies deformation in the normal direction, which resembles in a fluidic layer under compression or dilatation.

In this paper, we examined the dissipation behavior of ultrafine grained polycrystalline materials based on a continuum model coupled elastic deformation and viscous creep. We extended the understanding upon existing models [1,2,26-29,35] and revealed a second peak emerging at low frequencies in the loss modulus spectrum. The associated relaxation mechanisms of the two peaks are analyzed. The first peak is attributed to shear stress relaxation in GBs, and the second peak results from the relaxation of GB normal stress. For polycrystalline materials with viscous GBs, we obtain the expressions of critical frequencies when dissipation maximizes in terms of grain size: the first peak locates near a frequency inversely proportional to the grain size, and the second peak occurs near a frequency inversely proportional to the cube of grain size. The amplitude of the first peak decreases linear with grain size, while the amplitude of the second peak is independent of grain size.

It is important to note that the mechanisms for damping and the inherent frequency response are strongly governed by the structural makeup of materials [11,52]. We emphasize that the large aspect ratio of GBs (large $d / t$ or small $\phi$ ) in polycrystalline solids gives rise to two particular modes of deformation (sliding and squeezing) in the confined GB regions and is responsible for the two peaks. The two deformation modes lead to distinct dependence on grain size, yet they originate from the same microscopic mechanism - viscous flow in GBs.

Both GB elasticity and GB diffusion affect the occurrence of the two peaks phenomenon. Within the range: $0 \leq G_{R} / G \ll \phi^{3}$, $0 \leq R \ll \phi^{3}$, the loss modulus spectrum is unaffected by $G_{R} / G$ or $R$; for $G_{R} / G \sim \phi^{3}$, the second peak declines rapidly and move toward the first peak with increasing $G_{R} / G$, eventually vanishes when $G_{R} / G$ is significantly greater than $\phi^{3}$; while for $R \sim \phi^{3}$, with increasing 
$R$, the two peaks moves toward each other and the amplitude of the second peak does not decrease, and the two peaks eventually merge together when $R$ is large enough.

Due to the difficulty in measuring internal friction over a wide range of frequency, it was mostly measured as a function of temperature. The Arrhenius equation is then used to correlate temperature with viscosity or relaxation time of GBs via $\eta=$ $\eta_{0} \exp \left(Q / k_{\mathrm{B}} T\right)$, where $\eta_{0}$ is the referential viscosity and $Q$ is the activation energy of relaxation [2]. Berry and Pritchet [30] observed experimentally two relaxation peaks in the temperature spectrum of Al films. The measured activation energy is close to the activation energy of GB diffusion in pure $\mathrm{Al}$, about $0.55 \mathrm{eV}$.

With our theoretical analysis, we may obtain a quick estimate of $Q$. The grain size $d$ of the sample tested by Berry and Pritchet [30] is about $100 \mathrm{~nm}$, and the temperatures corresponding to the two peaks are $T_{1} \approx 285 \mathrm{~K}$ and $T_{2} \approx 600 \mathrm{~K}$, respectively. With GB thickness of $t=0.5 \mathrm{~nm}$, and using the scaling law of our work, the ratio of two viscosities at the two temperatures is $\frac{\eta_{1}^{\prime}}{\eta_{2}^{\prime}} \approx \phi^{-2}=$ $\left(\frac{d}{t}\right)^{2}$. From the Arrhenius equation, with $\eta_{1}^{\prime}=\eta_{0} \exp \left(\frac{Q}{k_{\mathrm{B}} T_{1}}\right)$ and $\eta_{2}^{\prime}=\eta_{0} \exp \left(\frac{Q}{k_{\mathrm{B}} T_{2}}\right)$, we have $Q=k_{\mathrm{B}} \log \left(\frac{d}{t}\right)^{2} /\left(\frac{1}{T_{1}}-\frac{1}{T_{2}}\right) \approx 0.5 \mathrm{eV}$. It may imply the two peaks observed by Berry and Pritchet [30] are qualitatively related to the two peaks reported in our analysis. We further note that a detailed comparison of experimental data with simulation results is still difficult given the broad distributions of grain size and GB viscosity in real materials, which usually lead to broadening peaks [27].

While the work is initially focused on the relaxation behavior of polycrystalline materials, similar viscoelastic relaxation systems are ubiquitous. For example, this phenomenon is found to be related to the restriction of the cell (elastic inclusion) to the relaxation modes of the extracellular matrix (viscoelastic) [53]. In a recent study, Wang et al. [54] achieved stretchable materials of high toughness and low hysteresis by a composite of two stretchable materials with large modulus contrast. The soft matrix shears greatly, spreading large stretch in a long segment of each fiber and leading to the high toughness. Another example is the tooth enamel which consists of parallel microscale and nanoscale ceramic columns or prisms interlaced with a soft protein matrix. This kind of combination and architecture of two different constituents enable tooth enamel to exhibit excellent resistance to both shortterm high-impact and long-term low-impact damage [55]. Inspire by this, Yeom et al. [56] reported an abiotic tooth enamel that exhibits a combination of stiffness, vibrational damping and low density. The unifying picture revealed based on the combination of hard grains and soft GB layer dissipation could be employed to many heterogeneous systems for their design for high damping performance.

\section{Declaration of Competing Interest}

The authors declare that they have no known competing financial interests or personal relationships that could have appeared to influence the work reported in this paper.

\section{Acknowledgments}

Y.W. acknowledges support from the NSFC Basic Science Center for 'Multiscale Problems in Nonlinear Mechanics' (No. 11988102) and (No. 11790291), the Strategic Priority Research Program of the Chinese Academy of Sciences (XDB22020200), and CAS Center for Excellence in Complex System Mechanics. Computation was supported by Beijing PARATERA Tech CO., Ltd.

\section{Appendix A}

Micromechanical based finite element modelling has been employed to obtain the dynamic properties of polycrystalline aggregates based on the mechanical response of the representative volume element (RVE). RVEs for both a 2D and a 3D model are constructed. Associated periodic boundary conditions can be developed for the RVEs.

\section{Hexagonal model}

In the 2D model, the polycrystalline aggregate composed of regular hexagons as shown in Fig. 1a is transversely isotropic in the $x-y$ plane and we can evaluate the effective complex shear modulus of the aggregate with arbitrary axial deformation modes. The RVE of the hexagonal model is outlined in the blue box in Fig. A.1a. Due to symmetries, only $1 / 4$ of the RVE is needed in the FE simulations as illustrated in Fig. A.1b. The symmetric boundary conditions are employed on the left and bottom boundary of the model. Nodes on the top edge are not allowed to move in the $y$ direction. Nodes on the right edge are prescribed with a time-dependent displacement in the $x$ direction, $u=u_{0} \sin (\omega t)$. The macroscopic strain is uniform strain in the $x$ direction, i.e., $\bar{\varepsilon}_{x x}=\varepsilon_{0} \sin (\omega t), \bar{\varepsilon}_{y y}=0$. Thereby, the maximum macroscopic shear strain is $\bar{\gamma}_{x y}=\bar{\varepsilon}_{x x}-\bar{\varepsilon}_{y y}=\varepsilon_{0} \sin (\omega t)$. By FE calculation, we can evaluate the resultant macroscopic stresses $\bar{\sigma}_{x x}$ and $\bar{\sigma}_{y y}$, which are also sinusoidal functions of time with the same frequency $\omega$ after a few cycles. Hence we can express the maximum macroscopic shear stress as $\bar{\tau}_{x y}=\left(\bar{\sigma}_{x x}-\bar{\sigma}_{y y}\right) / 2=\tau_{0} \sin (\omega t+\delta)$. Consequently, the effective complex shear modulus $G^{*}$ can be obtained by its definition:

$G^{*}=\hat{G} e^{i \delta}=G^{\prime}+i G^{\prime \prime}$

where, $\hat{G}=\tau_{0} / \varepsilon_{0}$ is the magnitude of the complex modulus which equals the ratio of the magnitude of $\bar{\tau}_{x y}$ to that of $\bar{\gamma}_{x y}$, and $\delta$ donates the phase angle of the complex modulus which equals the phase shift between $\bar{\tau}_{x y}$ and $\bar{\gamma}_{x y}, G^{\prime}=\hat{G} \cos (\delta)$ is the storage modulus and $G^{\prime \prime}=\hat{G} \sin (\delta)$ is the loss modulus.

The simulation results for the $2 \mathrm{D}$ hexagonal models with different $\phi$ and $G_{R} / G$ are shown in Fig. A.2.

\section{Wigner-Seitz model}

In the 3D model, the RVE is a Wigner-Seitz cell shown in Fig. A.3a, which is space-filling and spatially periodic. General periodic boundary conditions can be developed for the RVE under serval deformation modes [57].

\subsection{Uniaxial deformation along the $z$ direction}

In order to calculate the effective complex Young's modulus, the polycrystalline sample is subjected to uniaxial deformation in the $z$ direction. Hence, only $1 / 8$ of the RVE is considered in FE simulations due to the reflective symmetries (Fig. A.3b). The coordinate planes are symmetry planes and at the origin point $O_{1}$, $u\left(O_{1}\right)=v\left(O_{1}\right)=w\left(O_{1}\right)=0$. For points on the facet $S_{7}$, the displacement in the $z$ direction is prescribed:

$\boldsymbol{e}_{3} \cdot \boldsymbol{u}\left(S_{7}\right)=w$

where, $\boldsymbol{e}_{\boldsymbol{i}}$ are the unit basis vectors along the axis with $i=1,2,3$, $w$ denotes the applied displacement which is a sinusoidal function of time with frequency $\omega$. Now consider the boundary conditions on the facets $S_{1}$ and $S_{2}$. For a point $I_{1}(x, y, z)$ on the facet $S_{1}, I_{2}(\sqrt{2} h-x, y, h-z)$ is on the facet $S_{2}$, where $h$ is the height of the model in Fig. A.3b. To relate the displacement vector of $I_{1}$ to that of $I_{2}$, we need to take advantage of the periodicity and symmetry of the RVE. For point $I_{1}(x, y, z)$ and point $I_{1}^{\prime}(x,-y, z)$, which 
(a)

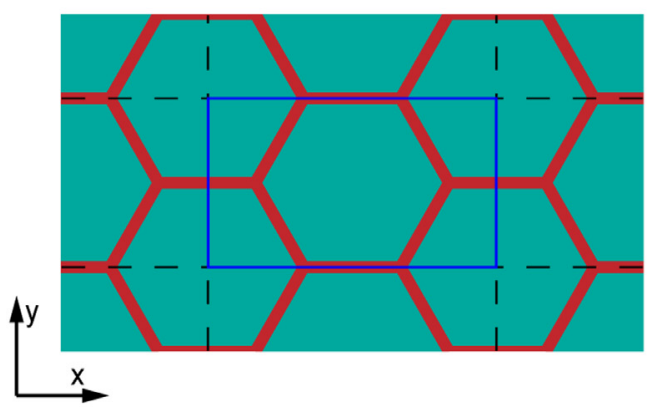

(b)

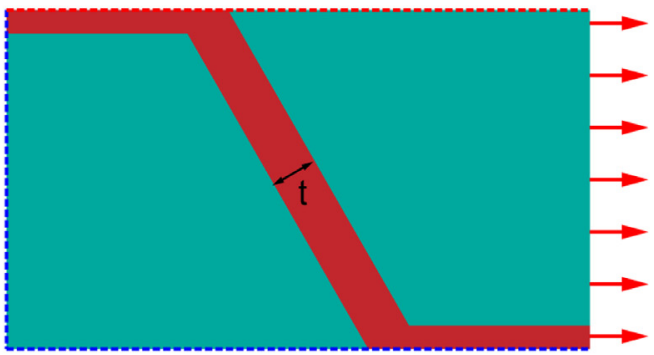

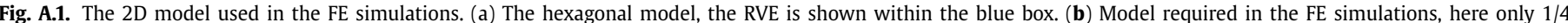
of the RVE is needed due to symmetries. (For interpretation of the references to color in this figure legend, the reader is referred to the web version of this article.)

(a)

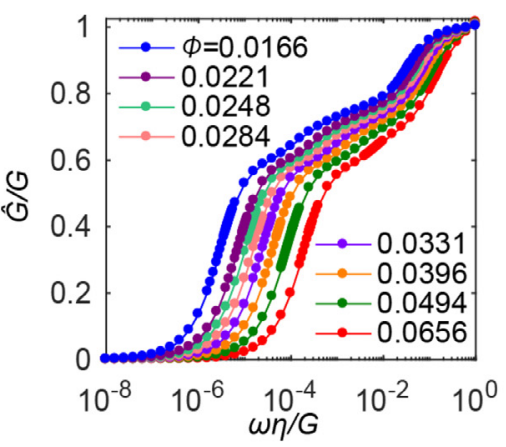

(d)

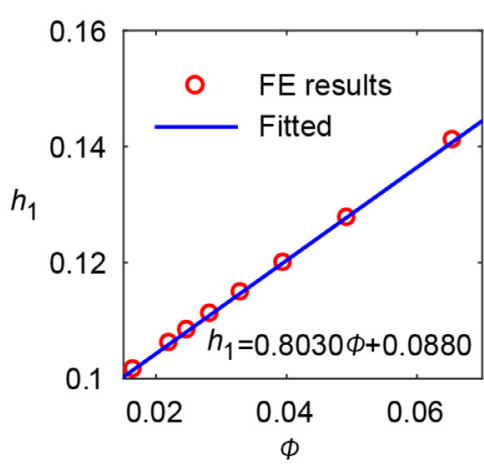

(b)

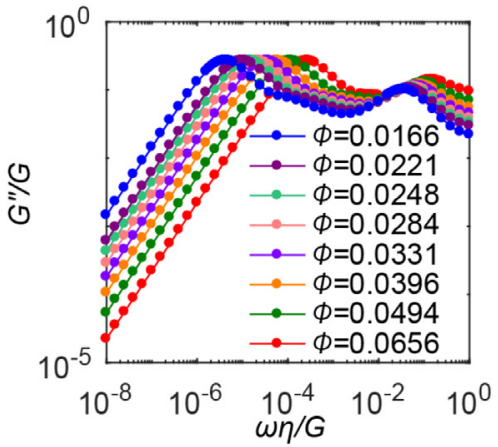

(e)

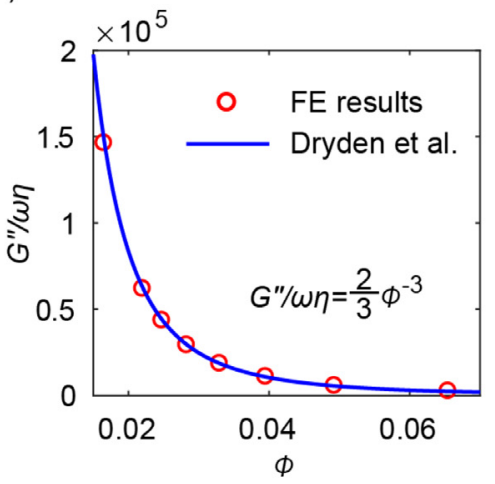

(c)

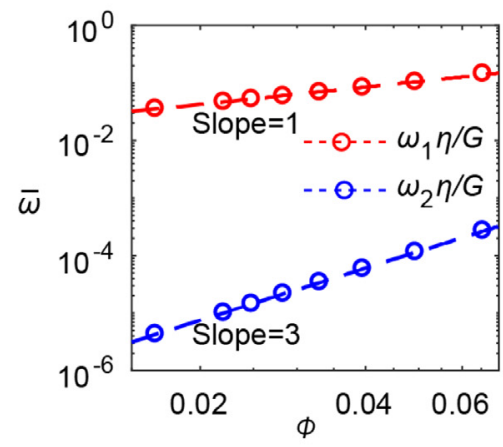

(f)

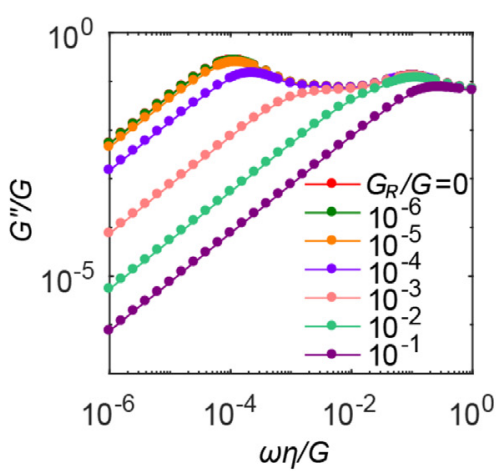

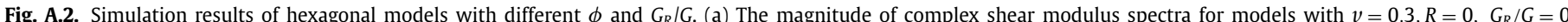

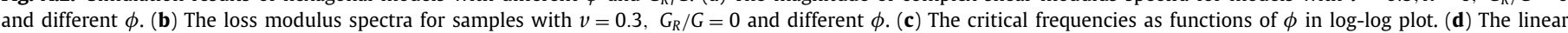

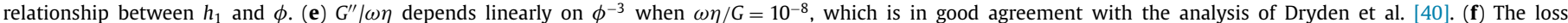
modulus spectra for samples with $\phi=0.0494, v=0.3, R=0$ and different $G_{R} / G$.

(a)

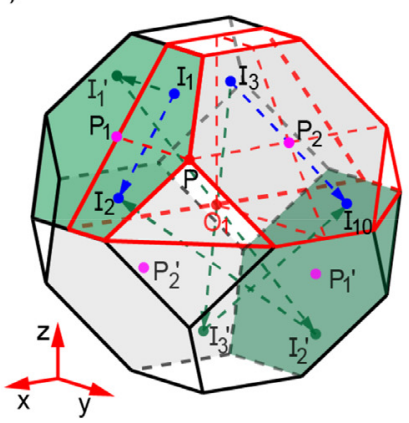

(b)

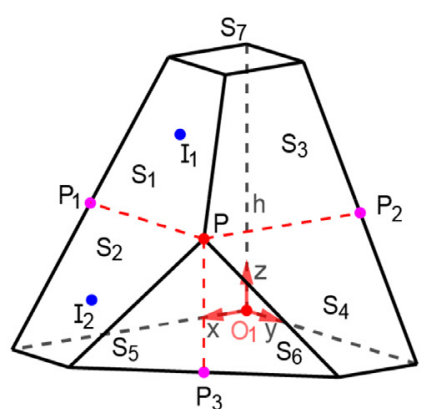

(c)

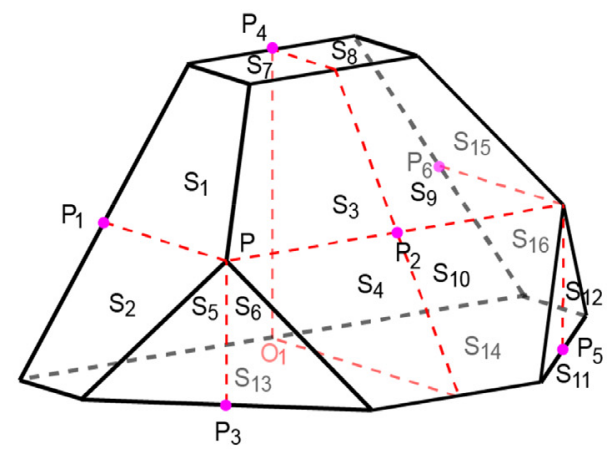

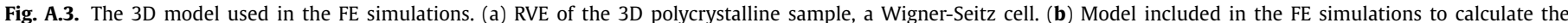
effective complex Young's modulus, here only $1 / 8$ of the RVE shown in (a) is needed. (c) 1/4 of the RVE is required to calculate the effective complex shear modulus. 
are reflectively symmetric with respect to $x-z$ plane, their displacements satisfy:

$\boldsymbol{u}\left(I_{1}^{\prime}\right)=\left(1-2 \boldsymbol{e}_{2} \otimes \boldsymbol{e}_{2}\right) \boldsymbol{u}\left(I_{1}\right)=\boldsymbol{Q}\left(\boldsymbol{e}_{2}\right) \boldsymbol{u}\left(I_{1}\right)$

where, 1 is the second-order identity tensor, $\otimes$ is the tensor product, and $\boldsymbol{Q}(\boldsymbol{n})$ is the reflection operator of the symmetry plane with normal direction $\boldsymbol{n}$. By central symmetry, for point $I_{1}^{\prime}(x,-y, z)$ and point $I_{2}^{\prime}(-x, y,-z)$, the formula relating their displacements is:

$\boldsymbol{u}\left(I_{1}^{\prime}\right)=-\boldsymbol{u}\left(I_{2}^{\prime}\right)$

The displacement vectors for $I_{2}^{\prime}(-x, y,-z)$ and $I_{2}(\sqrt{2} h-x, y, h-z)$ are related to displacement vectors of point $P_{1}\left(\frac{\sqrt{2}}{2} h, 0, \frac{h}{2}\right)$ and $P_{1}^{\prime}\left(-\frac{\sqrt{2}}{2} h, 0,-\frac{h}{2}\right)$ by:

$\mathbf{u}\left(I_{2}\right)-\mathbf{u}\left(I_{2}^{\prime}\right)=\mathbf{u}\left(P_{1}\right)-\mathbf{u}\left(P_{1}^{\prime}\right)$

Due to symmetry, we have:

$\mathbf{u}\left(P_{1}\right)=-\mathbf{u}\left(P_{1}^{\prime}\right)$

Therefore, by combining Eqs. (A.3) - (A.6), we obtain:

$\boldsymbol{u}\left(I_{2}\right)+\boldsymbol{Q}\left(\boldsymbol{e}_{2}\right) \boldsymbol{u}\left(I_{1}\right)=2 \boldsymbol{u}\left(P_{1}\right)$

Eq. (A.7) expresses the relationship between the displacement vectors of two points on the facets $S_{1}$ and $S_{2}$ which are reflectively symmetric with respect to line $P P_{1}$. We rewrite it as follows:

$\boldsymbol{u}\left(S_{2}\right)+\boldsymbol{Q}\left(\boldsymbol{e}_{2}\right) \boldsymbol{u}\left(S_{1}\right)=2 \boldsymbol{u}\left(P_{1}\right)$

Similarly, we can obtain:

$\boldsymbol{u}\left(S_{4}\right)+\boldsymbol{Q}\left(\boldsymbol{e}_{1}\right) \boldsymbol{u}\left(S_{3}\right)=2 \boldsymbol{u}\left(P_{2}\right)$

$\boldsymbol{u}\left(S_{5}\right)+\boldsymbol{Q}\left(\boldsymbol{e}_{3}\right) \boldsymbol{u}\left(S_{6}\right)=2 \boldsymbol{u}\left(P_{3}\right)$

Due to periodicity, $\boldsymbol{u}\left(P_{1}\right)-\boldsymbol{u}\left(O_{1}\right)=\boldsymbol{u}\left(P^{\prime}{ }_{1}\right)-\boldsymbol{u}\left(O_{1}^{\prime}\right)$, where point $O_{1}^{\prime}(-\sqrt{2} h, 0,-h)$ is at the center of a neighboring grain. In the case of uniaxial deformation, $w\left(O_{1}\right)-w\left(O_{1}^{\prime}\right)=w$, thus $w\left(P_{1}\right)=\frac{w}{2}$. Consequently, we have $w\left(P_{1}\right)=w\left(P_{2}\right)=\frac{w}{2}, v\left(P_{1}\right)=u\left(P_{2}\right)=w\left(P_{3}\right)=0$, $u\left(P_{1}\right), v\left(P_{2}\right), u\left(P_{3}\right)$ and $v\left(P_{3}\right)$ are left un-prescribed. The symmetry conditions on the coordinate planes and Eqs. (A.2), (A.8) constitute the boundary conditions of the model under uniaxial deformation.

\subsection{Simple shear in the $x-z$ plane along the $x$ direction}

The polycrystalline sample is subjected to macroscopic simple shear deformation in the $x-z$ plane along the $x$ direction to calculate the effective complex shear modulus of the Wigner-Seitz model. In this case, $1 / 4$ of the RVE as shown in Fig. A.3c is needed in the simulations. Note that the $x-z$ plane is a plane of symmetric as in the case of uniaxial deformation, the boundary condition concerning points on the facets $S_{1}$ and $S_{2}$ is still valid. We may repeat it as follows:

$\boldsymbol{u}\left(S_{2}\right)+\boldsymbol{Q}\left(\boldsymbol{e}_{2}\right) \boldsymbol{u}\left(S_{1}\right)=2 \boldsymbol{u}\left(P_{1}\right)$

Similarly, we have:

$$
\begin{aligned}
& \boldsymbol{u}\left(S_{16}\right)+\boldsymbol{Q}\left(\boldsymbol{e}_{2}\right) \boldsymbol{u}\left(S_{15}\right)=2 \boldsymbol{u}\left(P_{6}\right) \\
& u\left(S_{8}\right)+Q\left(e_{2}\right) u\left(S_{7}\right)=2 u\left(P_{4}\right) \\
& \boldsymbol{u}\left(S_{6}\right)-\boldsymbol{Q}\left(\boldsymbol{e}_{2}\right) \boldsymbol{u}\left(S_{12}\right)=2 \boldsymbol{u}\left(P_{3}\right) \\
& \boldsymbol{u}\left(S_{11}\right)-\boldsymbol{Q}\left(\boldsymbol{e}_{2}\right) \boldsymbol{u}\left(S_{5}\right)=2 \boldsymbol{u}\left(P_{5}\right) \\
& \boldsymbol{u}\left(S_{14}\right)+\boldsymbol{Q}\left(\boldsymbol{e}_{2}\right) \boldsymbol{u}\left(S_{13}\right)=0
\end{aligned}
$$

Since the $x-y$ plane is a plane of antisymmetric for the case of simple shear, the displacement vectors of points on the facets $S_{3}$ and $S_{10}$ are related:

$\boldsymbol{u}\left(S_{3}\right)+\boldsymbol{u}\left(S_{10}\right)=2 \boldsymbol{u}\left(P_{2}\right)$

Similarly, we can obtain:

$\boldsymbol{u}\left(S_{4}\right)+\boldsymbol{u}\left(S_{9}\right)=2 \boldsymbol{u}\left(P_{2}\right)$

The displacement vector of point $P_{i}$ in Eq. (A9) is

$\boldsymbol{u}\left(P_{i}\right)=(\boldsymbol{F}-1) \boldsymbol{p}\left(P_{i}\right)$

where, $\boldsymbol{p}\left(P_{i}\right)$ is the coordinate of the reference point $P_{i}(i=$ $1,2 \ldots 6)$. $F$ is the macroscopic deformation gradient, and for the case of simple shear:

$\boldsymbol{F}=\left[\begin{array}{lll}1 & 0 & \gamma \\ 0 & 1 & 0 \\ 0 & 0 & 1\end{array}\right]$

where, $\gamma$ is the applied macroscopic shear strain.

The symmetric boundary conditions on the $x-z$ plane, antisymmetric boundary conditions on the $x-y$ plane and Eq. (A.9) constitute the boundary conditions of the model in the case of simple shear.

\section{Appendix B}

By definition, anelastic is a subset of viscoelastic behavior and it means completely recoverable behavior [58]. Anelastic materials have unique equilibrium configurations and are fully recoverable upon removal of a transient perturbation. In this paper, while microscopic GB mechanism for internal dissipation is nonrecoverable, the macroscopic behavior of a polycrystalline aggregate may be anelastic.

In Fig. B.1, we show the stress-strain curves for the 2D hexagonal models with $\phi=0.0494$ and 3D Wigner-Seitz models with $\phi=0.0902$. The load-time profiles are shown in the insets. As it shows, hysteresis in the stress-strain curves is rate-dependent. Within a loading cycle, the viscoelastic constitutive equation gives rise to fully recoverable deformation at sufficiently long dwelling time as long as $G_{R}>0$. In order to have an anelastic behavior, a restoring force is necessary. In our polycrystalline models shown in Fig. 1, considering the GBs are continuous and the grains are isolated, we need to set $G_{R}>0$. In the models of Zener [1], Ghahremani [26] and Lee and Morris [27], the constrains assigned in the normal direction of GBs serve as the restoring force and lead to a completely recoverable behavior. For the case of $G_{R}=0$, if the elastic grains are continuous, then the macroscopic behavior may be fully recoverable. We use a simple structure, as shown in Fig. B.2a, to demonstrate the macroscopically recoverable deformation (Fig. B.2c and d) and the two peaks in loss modulus spectrum (Fig. B.2b). Hence, both the microscopic structures and GB elasticity govern the recoverable behavior of the materials.

Table B.1

Material properties and parameters used in the simulations.

\begin{tabular}{lll}
\hline Properties & Symbol & Value \\
\hline Shear modulus of grain interior & $G$ & $139 \mathrm{GPa}$ \\
Bulk modulus of grain interior and GB & $K$ & $301 \mathrm{GPa}$ \\
Shear modulus of GB & $G_{R}$ & $0.0139 \mathrm{GPa}$ \\
Viscosity of GB & $\eta$ & $1 \mathrm{GPa} \cdot \mathrm{s}$ \\
GB diffusivity & $D_{0}$ & 0 \\
\hline
\end{tabular}


(a)

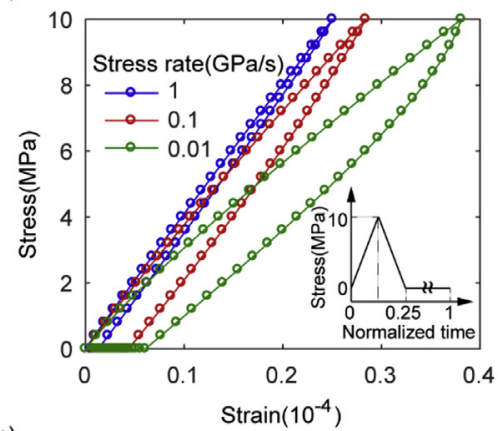

(c)

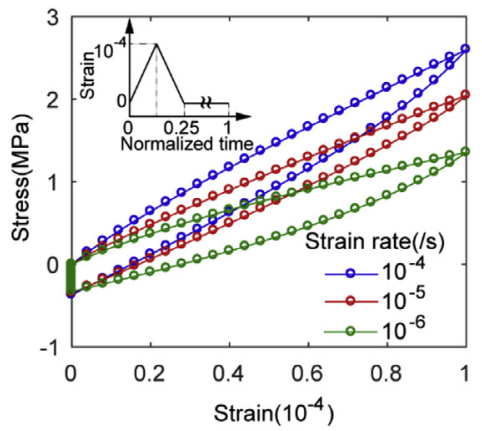

(b)

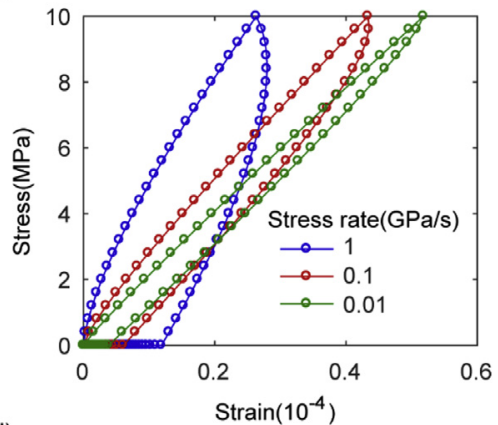

(d)

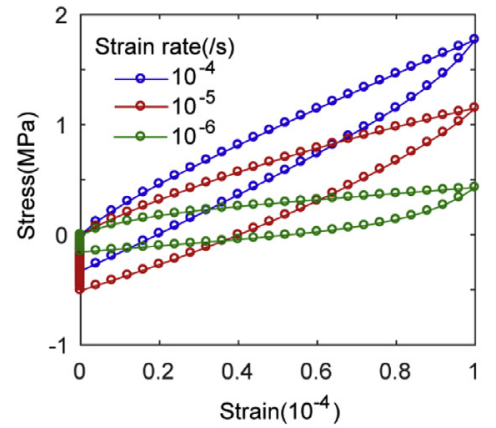

Fig. B.1. Stress-strain curves for the 2D and 3D models subjected to macroscopic uniaxial tension. Stress controlled loading for (a) 2D models and (b) 3D samples. Strain controlled loading for (c) 2D models and (d) 3D samples. The load-time profile for stress loading conditions is shown in the inset of (a), and that of strain loading is shown in the inset of $(\mathbf{c})$. The material parameters used for the calculations are given in Table B.1.

(a)

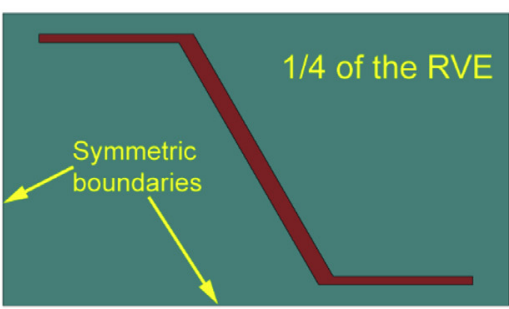

(c)

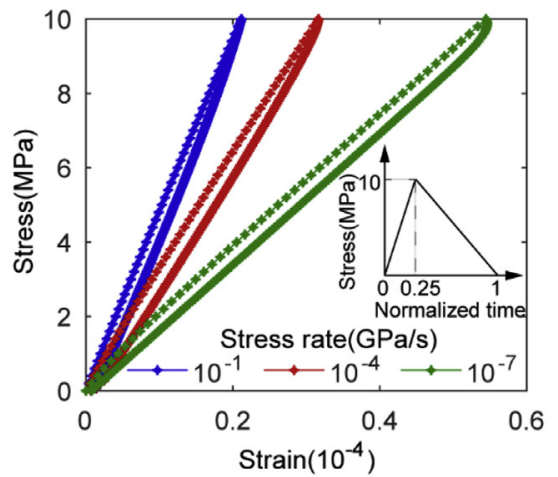

(b)

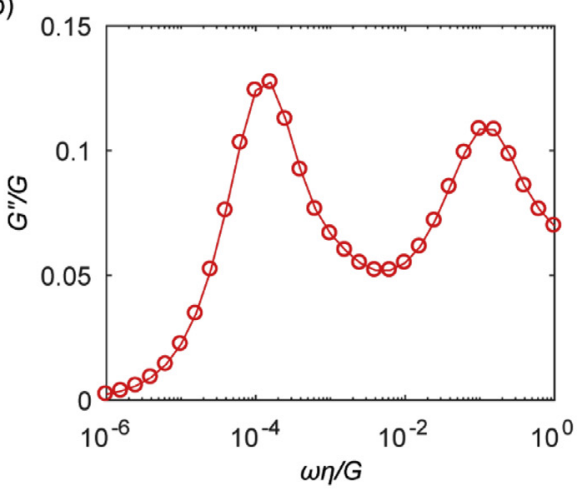

(d)

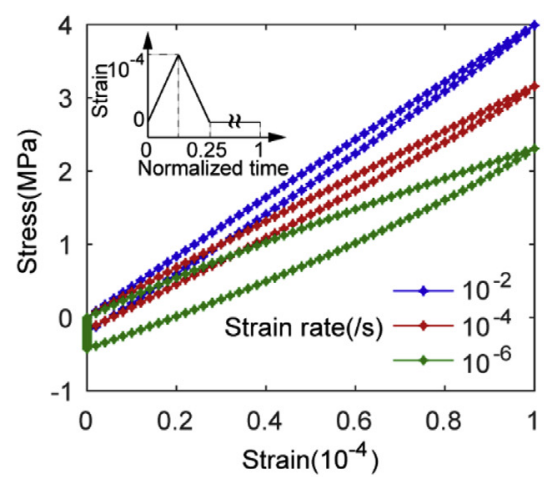

Fig. B.2. Demonstration of two peaks for loss modulus spectrum and the recoverable deformation when GB elasticity is neglected, i.e., $G_{R}=0$. (a) The micromechanical model with grain interiors in green and GBs in red. (b) Loss modulus spectrum for the model shown in (a). Stress-strain curves for the model under macroscopic uniaxial tension with stress controlled loading in (c) and strain controlled loading in (d). The load-time profiles are shown in the insets. The material parameters used for the calculations are given in Table B.1, except that $G_{R}=0$ is used here. (For interpretation of the references to color in this figure legend, the reader is referred to the web version of this article.) 


\section{References}

[1] C. Zener, Theory of the elasticity of polycrystals with viscous grain boundaries, Phys. Rev. 60 (1941) 906-908.

[2] T.-S. Kê, Experimental evidence of the viscous behavior of grain boundaries in metals, Phys. Rev. 71 (1947) 533-546.

[3] B. Cao, R. Schaller, W. Benoit, F. Cosandey, Internal friction associated with grain boundaries in Ni-Cr alloys, J. Alloys Compd. 211-212 (1994) 118-123.

[4] C. Dae-han, K. Hyungsub, W.D. Nix, Anelasticity and damping of thin aluminum films on silicon substrates, J. Microelectromech. Syst. 13 (2004) 230-237.

[5] H. Watanabe, T. Mukai, M. Sugioka, K. Ishikawa, Elastic and damping properties from room temperature to $673 \mathrm{~K}$ in an AZ31 magnesium alloy, Scr. Mater. 51 (2004) 291-295.

[6] X.S. Hu, Y.K. Zhang, M.Y. Zheng, K. Wu, A study of damping capacities in pure $\mathrm{Mg}$ and Mg-Ni alloys, Scr. Mater. 52 (2005) 1141-1145.

[7] E.F. Dudarev, G.P. Pochivalova, Y.R. Kolobov, E.V. Naydenkin, O.A. Kashin, Diffusion-controlled true grain-boundary sliding in nanostructured metals and alloys, Mater. Sci. Eng. A 503 (2009) 58-61.

[8] Q.P. Kong, W.B. Jiang, Y. Shi, P. Cui, Q.F. Fang, M. Winning, Grain boundary internal friction in bicrystals with different misorientations, Mater. Sci. Eng. A 521-522 (2009) 128-133.

[9] A.-K. Maier, D. Mari, I. Tkalcec, R. Schaller, Theoretical modelling of grain boundary anelastic relaxations, Acta Mater. 74 (2014) 132-140.

[10] J.I. Rojas, B. Venkata Siva, K.L. Sahoo, D. Crespo, Viscoelastic behavior of a novel aluminum metal matrix composite and comparison with pure aluminum, aluminum alloys, and a composite made of $\mathrm{Al}-\mathrm{Mg}-\mathrm{Si}$ alloy reinforced with $\mathrm{SiC}$ particles, J. Alloys Compd. 744 (2018) 445-452.

[11] M.S. Blanter, I.S. Golovin, H. Neuhauser, H.R. Sinninng, Internal Friction in Metallic Materials: A Handbook, Springer-Verlag, Berlin Heidelberg, 2007.

[12] J.L. Snoek, Effect of small quantities of carbon and nitrogen on the elastic and plastic properties of iron, Physica 8 (1941) 711-733.

[13] G. Fantozzi, C. Esnouf, W. Benoit, I.G. Ritchie, Internal friction and microdeformation due to the intrinsic properties of dislocations: The Bordoni relaxation, Prog. Mater. Sci. 27 (1982) 311-451.

[14] J.C. Ye, J. Lu, C.T. Liu, Q. Wang, Y. Yang, Atomistic free-volume zones and inelastic deformation of metallic glasses, Nat. Mater. 9 (2010) 619-623.

[15] W.H. Wang, The elastic properties, elastic models and elastic perspectives of metallic glasses, Prog. Mater. Sci. 57 (2012) 487-656.

[16] Q. Wang, S.T. Zhang, Y. Yang, Y.D. Dong, C.T. Liu, J. Lu, Unusual fast secondary relaxation in metallic glass, Nat. Commun. 6 (2015) 7876.

[17] J.D. Ferry, Viscoelastic Properties of Polymers, Third Edition, John Wiley \& Sons, New York, 1980.

[18] G. Tsagaropoulos, A. Eisenberg, Dynamic mechanical study of the factors affecting the two glass transition behavior of filled polymers. similarities and differences with random ionomers, Macromolecules 28 (1995) 6067-6077.

[19] D. Turcotte, G. Schubert, Geodynamics, Third Edition, Cambridge University Press, Cambridge, 2014.

[20] K. Wang, Y. Hu, J. He, Deformation cycles of subduction earthquakes in a viscoelastic Earth, Nature 484 (2012) 327-332.

[21] I. Jackson, J.D. Fitz Gerald, U.H. Faul, B.H. Tan, Grain-size-sensitive seismic wave attenuation in polycrystalline olivine, J. Geophys. Res. Solid Earth 107 (2002) 2360-2375.

[22] M. Sundberg, R.F. Cooper, A composite viscoelastic model for incorporating grain boundary sliding and transient diffusion creep; correlating creep and attenuation responses for materials with a fine grain size, Philos. Mag. 90 (2010) 2817-2840.

[23] S.-I. Karato, T. Olugboji, J. Park, Mechanisms and geologic significance of the mid-lithosphere discontinuity in the continents, Nat. Geosci. 8 (2015) 509-514.

[24] S.-I. Karato, Importance of anelasticity in the interpretation of seismic tomography, Geophys. Res. Lett 20 (1993) 1623-1626.

[25] R.F. Cooper, Seismic wave attenuation: energy dissipation in viscoelastic crystalline solids, Rev. Mineral. Geochem. 51 (2002) 253-290.

[26] F. Ghahremani, Effect of grain boundary sliding on anelasticity of polycrystals, Int. J. Solids Struct. 16 (1980) 825-845.

[27] L.C. Lee, S.J.S. Morris, Anelasticity and grain boundary sliding, Proc. Math. Phys. Eng. Sci. 466 (2010) 2651-2671.

[28] S.J.S. Morris, I. Jackson, Diffusionally assisted grain-boundary sliding and viscoelasticity of polycrystals, J. Mech. Phys. Solids 57 (2009) 744-761.
[29] L.C. Lee, S.J.S. Morris, J. Wilkening, Stress concentrations, diffusionally accommodated grain boundary sliding and the viscoelasticity of polycrystals, Proc. Math. Phys. Eng. Sci. 467 (2011) 1624-1644.

[30] B.S. Berry, W.C. Pritchet, Defect studies of thin layers by the vibrating-reed technique, J. Phys. Colloques 42 (1981) C5-1111-C5-1122.

[31] B. Cai, Q.P. Kong, P. Cui, H.T. Cong, X.K. Sun, Internal friction of nanocrystalline aluminum prepared by plasma evaporation and compaction, Scr. Mater. 44 (2001) 1043-1048.

[32] Z. Yang, J. Chen, L. He, H. Cong, H. Ye, Microstructure and grain boundary relaxation in ultrafine-grained Al/Al oxide composites, Acta Mater. 57 (2009) 3633-3644.

[33] J.S. Tello, A.F. Bower, E. Chason, B.W. Sheldon, Kinetic model of stress evolution during coalescence and growth of polycrystalline thin films, Phys. Rev. Lett. 98 (2007) 216104.

[34] Z. Suo, A continuum theory that couples creep and self-diffusion, J. Appl. Mech 71 (5) (2004) 646-651.

[35] J.B. Walsh, New analysis of attenuation in partially melted rock, J. Geophys. Res. 74 (1969) 4333-4337.

[36] R. Raj, M. Ashby, On grain boundary sliding and diffusional creep, Metall. Trans. 2 (1971) 1113-1127.

[37] Dassault Systemes Simulia Corp., Abaqus 3DEXPERIENCE R2017x, analysis user's guide (2017).

[38] A.F. Bower, Applied Mechanics of Solids, CRC press, Boca Raton, FL, 2009.

[39] Y. Wei, A.F. Bower, H. Gao, Recoverable creep deformation and transient local stress concentration due to heterogeneous grain-boundary diffusion and sliding in polycrystalline solids, J. Mech. Phys. Solids 56 (4) (2008) 1460-1483.

[40] J.R. Dryden, D. Kucerovsky, D.S. Wilkinson, D.F. Watt, Creep deformation due to a viscous grain boundary phase, Acta Metall. 37 (1989) 2007-2015.

[41] J.C. Jaeger, Elasticity, Fracture and Flow: With Engineering and Geological Applications, Third Edition, Chapman and Hall, London, 1969.

[42] R.J. O'Connell, B. Budiansky, Viscoelastic properties of fluid-saturated cracked solids, J. Geophys. Res. 82 (1977) 5719-5735.

[43] G. Mavko, A. Nur, Melt squirt in the asthenosphere, J. Geophys. Res. 80 (1975) $1444-1448$.

[44] E.H. Lee, Stress analysis in viscoelastic bodies, Q. Appl. Math. 13 (1955) 183-190.

[45] J.B. Walsh, Attenuation in partially melted material, J. Geophys. Res. 73 (1968) 2209-2216.

[46] R.L. Coble, A model for boundary diffusion controlled creep in polycrystalline materials, J. Appl. Phys. 34 (6) (1963) 1679-1682.

[47] H. Frost, M. Ashby, Deformation Mechanism Maps: The Plasticity and Creep of Metals and Ceramic, Pergamon Press, Oxford, 1982.

[48] H. Gleiter, Nanocrystalline materials, Prog. Mater. Sci. 33 (4) (1989) 223-315.

[49] J. Schiøtz, F.D. Di Tolla, K.W. Jacobsen, Softening of nanocrystalline metals at very small grain sizes, Nature 391 (6667) (1998) 561-563.

[50] Y. Wei, A.F. Bower, H. Gao, Enhanced strain-rate sensitivity in fcc nanocrystals due to grain-boundary diffusion and sliding, Acta Mater. 56 (8) (2008) $1741-1752$.

[51] D. Wolf, V. Yamakov, S.R. Phillpot, A. Mukherjee, H. Gleiter, Deformation of nanocrystalline materials by molecular-dynamics simulation: relationship to experiments? Acta Mater. 53 (1) (2005) 1-40.

[52] R. Ranganathan, Y. Shi, P. Keblinski, Frequency-dependent mechanical damping in alloys, Phys. Rev. B 95 (21) (2017) 214112.

[53] A.S.G. van Oosten, X. Chen, L. Chin, K. Cruz, A.E. Patteson, K. Pogoda, V.B. Shenoy, P.A. Janmey, Emergence of tissue-like mechanics from fibrous networks confined by close-packed cells, Nature 573 (2019) 96-101.

[54] Z. Wang, C. Xiang, X. Yao, P. Le Floch, J. Mendez, Z. Suo, Stretchable materials of high toughness and low hysteresis, Pro. Nat. Acad. Sci. U.S.A 116 (2019) 5967-5972.

[55] H.D. Espinosa, R. Soler-Crespo, Lessons from tooth enamel, Nature 543 (2017) $42-43$.

[56] B. Yeom, T. Sain, N. Lacevic, D. Bukharina, S.-H. Cha, A.M. Waas, E.M. Arruda, N.A. Kotov, Abiotic tooth enamel, Nature 543 (2017) 95-98.

[57] M. Danielsson, D.M. Parks, M.C. Boyce, Three-dimensional micromechanical modeling of voided polymeric materials, J. Mech. Phys. Solids 50 (2002) 351-379.

[58] R. Lakes, Viscoelastic Materials, Cambridge University Press, Cambridge, 2009. 